\title{
iPTF17cw: An Engine-driven Supernova Candidate Discovered Independent of a Gamma-Ray Trigger
}

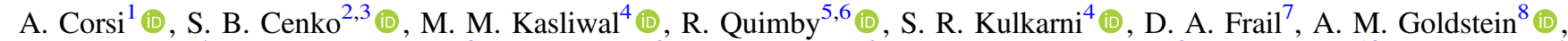
N. Blagorodnova ${ }^{4}$ (D) V. Connaughton ${ }^{8}$, D. A. Perley ${ }^{9}$ (D) , L. P. Singer $^{2}$ (D), C. M. Copperwheat ${ }^{9}$, C. Fremling ${ }^{10}$ (iD), T. Kupfer ${ }^{4}$ (iD),


J. Sollerman ${ }^{10}$ (D), E. Troja ${ }^{2,11}$ (D), and S. Veilleux ${ }^{3,11}$ (D)

${ }^{1}$ Department of Physics and Astronomy, Texas Tech University, Box 1051, Lubbock, TX 79409-1051, USA; alessandra.corsi@ttu.edu 2 Astrophysics Science Division, NASA Goddard Space Flight Center, Greenbelt, MD 20771, USA 3 Joint Space-Science Institute, University of Maryland, College Park, MD 20742, USA

${ }_{5}^{4}$ Division of Physics, Mathematics, and Astronomy, California Institute of Technology, Pasadena, CA 91125, USA

${ }^{5}$ Department of Astronomy/Mount Laguna Observatory, San Diego State University, San Diego, CA 92182, USA

${ }^{6}$ Kavli IPMU (WPI), UTIAS, The University of Tokyo, Kashiwa, Chiba 277-8583, Japan

${ }^{7}$ National Radio Astronomy Observatory, P.O. Box O, Socorro, NM 87801, USA

${ }^{8}$ Universities Space Research Association, NSSTC, 320 Sparkman Drive, Huntsville, AL 35805, USA

${ }^{9}$ Astrophysics Research Institute, Liverpool John Moores University, IC2, Liverpool Science Park, 146 Brownlow Hill, Liverpool, L3 5RF, UK

${ }^{10}$ Oskar Klein Centre, Department of Astronomy, Stockholm University, Albanova University Centre, SE-106 91 Stockholm, Sweden

${ }^{11}$ Department of Astronomy, University of Maryland, College Park, MD 20742, USA

12 Astrophysics Office, ZP12, NASA/Marshall Space Flight Center, Huntsville, AL 35812, USA

Received 2017 May 15; revised 2017 August 8; accepted 2017 August 9; published 2017 September 20

\begin{abstract}
We present the discovery, classification, and radio-to-X-ray follow-up observations of iPTF17cw, a broad-lined (BL) type Ic supernova (SN) discovered by the intermediate Palomar Transient Factory (iPTF). Although it is unrelated to the gravitational wave trigger, this SN was discovered as a happy by-product of the extensive observational campaign dedicated to the follow-up of Advanced LIGO event GW 170104. The spectroscopic properties and inferred peak bolometric luminosity of iPTF17cw are most similar to the gamma-ray-burst (GRB)associated SN, SN 1998bw, while the shape of the $r$-band light curve is most similar to that of the relativistic SN, SN 2009bb. Karl G. Jansky Very Large Array (VLA) observations of the iPTF17cw field reveal a radio counterpart $\approx 10$ times less luminous than SN 1998bw, and with a peak radio luminosity comparable to that of SN 2006aj/ GRB 060218 and SN 2010bh/GRB 100316D. Our radio observations of iPTF17cw imply a relativistically expanding outflow. However, further late-time observations with the VLA in its most extended configuration are needed to confirm fading of the iPTF17cw radio counterpart at all frequencies. X-ray observations carried out with Chandra reveal the presence of an X-ray counterpart with a luminosity similar to that of SN2010bh/ GRB 100316D. Searching the Fermi catalog for possible $\gamma$-rays reveals that GRB 161228B is spatially and temporally compatible with iPTF17cw. The similarity to SN 1998bw and SN 2009bb, the radio and X-ray detections, and the potential association with GRB 161228B all point to iPTF17cw being a new candidate member of the rare sample of optically discovered engine-driven BL-Ic SNe associated with relativistic ejecta.
\end{abstract}

Key words: gamma-ray burst: individual (GRB 161228B) - gravitational waves - supernovae: individual (iPTF17cw)

\section{Introduction}

Almost 20 years after the discovery of an association between the radio-loud, broad-lined (BL), type Ic supernova (SN), SN 1998bw, and the low-luminosity, long gamma-ray burst (GRB), GRB 980425 (Galama et al. 1998; Kulkarni et al. 1998), we still have only a handful of GRB-associated (or engine-driven) SNe with radio light curves (Berger et al. 2003b; Soderberg et al. 2004, 2006a; Frail et al. 2005; Margutti et al. 2013; Perley et al. 2014; Singer et al. 2015). While the link between long GRBs and BL-Ic SNe is well established (Woosley \& Bloom 2006), it remains unclear what makes some BL-Ic SNe launch ultra-relativistic jets (GRBs). Radio observations are key to shedding light on this open question because radio probes the fastest moving ejecta and is unaffected by beaming effects that preclude detection of $\mathrm{X}$-rays and $\gamma$-rays from the off-axis GRBs that may drive some BL-Ic SNe.

\footnotetext{
${ }^{13}$ NASA Postdoctoral Fellow.
}

The discovery of explosions such as SN 2009bb (Soderberg et al. 2010), a relativistic BL-Ic SN without a detected GRB, has also prompted the intriguing question of whether there exists a population of events with properties in between that of "ordinary" BL-Ic SNe and GRBs (ultra-relativistic jets). Radio, by being effective at also probing mildly relativistic explosions, can be used as a tool to search for these events "in the gap" (see also Ofek et al. 2007; Milisavljevic et al. 2015).

Over the last decade, the above considerations have motivated extensive searches for radio emission from type Ib/c SNe (e.g., Berger et al. 2003a; Soderberg et al. 2006b; Bietenholz et al. 2014), which have constrained the fraction of $\mathrm{Ib} / \mathrm{c}$ SNe associated with GRBs to $\lesssim 1 \%-3 \%$. Most of these studies, however, have long been limited by the very small number of BL-Ic SNe available to the community (Berger et al. 2003a; Chomiuk \& Soderberg 2010; Soderberg et al. 2010; Corsi et al. 2011; Soderberg \& Chomiuk 2011; Drake et al. 2013; Kamble \& Soderberg 2013; Salas et al. 2013; Corsi et al. 2014; Chakraborti et al. 2015; Milisavljevic et al. 2015). BL-Ic 


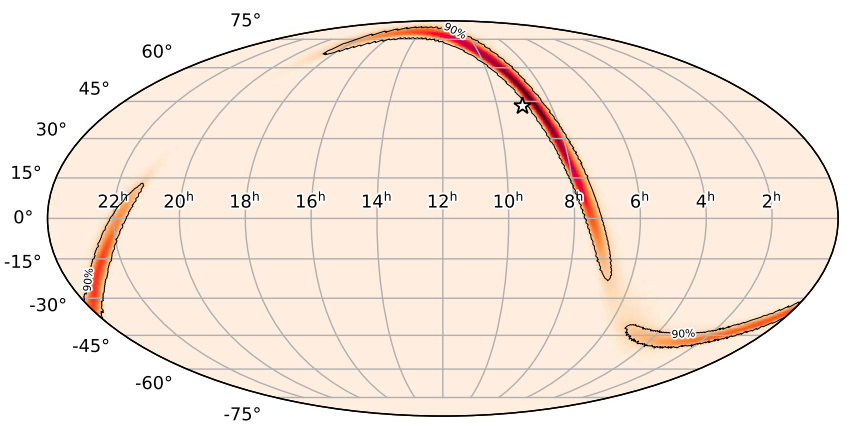

Figure 1. Position of iPTF17cw superimposed on the final LIGO localization (LIGO Scientific Collaboration \& Virgo Collaboration 2017c). The color represents the probability density. A black contour is drawn around the LIGO $90 \%$ credible region. Because of the binary black hole nature of GW 170104 (Abbott et al. 2017), and given that iPTF17cw falls just outside of the $90 \%$ localization area of GW 170104, we rule out any association between iPTF17cw and GW 170104.

SNe are the only type of SNe observationally linked to GRBs (Woosley \& Bloom 2006), but not all BL-Ic SNe are enginedriven. Recently, thanks to the much-increased rate of BL-Ic discoveries enabled by the Palomar Transient Factory (PTF and its successor, the intermediate Palomar Transient Factory (iPTF); Law et al. 2009; Rau et al. 2009), we have constrained the fraction of BL-Ic SNe as relativistic as (and observationally similar to) $\mathrm{SN} 1998 \mathrm{bw}$ to $\lesssim 41 \%$ of the BL-Ic population (99.865\% confidence; Corsi et al. 2016).

Based on the above limits, it is clear that relativistic BL-Ic $\mathrm{SNe}$ are rare events, and the number of BL-Ic SNe with relativistic ejecta discovered independently of a $\gamma$-ray trigger remains extremely small. Here, we present the discovery of a candidate relativistic BL-Ic SN, dubbed iPTF17cw. This event was discovered by the iPTF during an extensive follow-up campaign of the Advanced LIGO event GW 170104 (Corsi et al. 2017; Evans et al. 2017; Kasliwal et al. 2017; Kupfer et al. 2017; LIGO Scientific Collaboration \& Virgo Collaboration 2017a, 2017b, 2017c). Because of the binary black hole nature of GW 170104 (Abbott et al. 2017), and given that iPTF17cw falls just outside of the $90 \%$ localization area of GW 170104 (Figure 1), we rule out any association between iPTF17cw and GW 170104.

In what follows, we describe our data set (Section 2), analyze our multi-wavelength observations (Section 3), and show that iPTF17cw adds one more candidate to the sample of BL-Ic $\mathrm{SNe}$ from which we have ever detected radio emission (indicative of relativistic expansion) independent of a $\gamma$-ray trigger. iPTF17cw also adds one more event to the more general class of relativistic optical transients discovered independently of a high-energy trigger (Cenko et al. 2013, 2015). An after-the-fact search for $\gamma$-rays reveals that iPTF17cw may be associated with GRB 161228B. In our conclusion (Section 4), we stress how further late-time radio observations of the iPTF17cw field are needed to securely confirm its relativistic nature.

\section{Panchromatic Observations}

\subsection{SN Optical Photometry}

We discovered iPTF17cw on 2017 January 7 UT in an $R$-band image taken with the $7.8 \mathrm{deg}^{2}$ wide camera on the Palomar Samuel Oschin 48 inch Schmidt Telescope (P48), which is routinely used by the iPTF (Law et al. 2009; Cao et al. 2016;

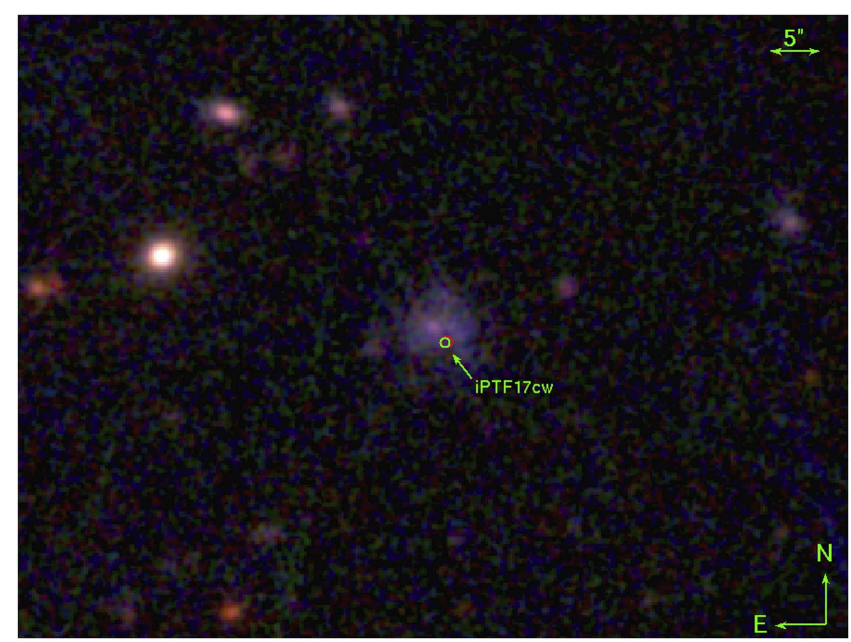

Figure 2. Pan-STARRS1 (Kaiser et al. 2010; Chambers et al. 2016) preimaging of the host galaxy of iPTF $17 \mathrm{cw}$, with the location of the optical transient superimposed $\left(0.0^{\prime \prime} 5\right.$ error circle shown in green, which also contains the position of the radio counterpart). We also show the error circle of the $\mathrm{X}$-ray counterpart to iPTF17cw (red). The host is blue, extended, and diffuse, with a reddish central concentration. The optical position (as well as radio and $\mathrm{X}$-ray positions) is (are) significantly offset (by $\approx 1$ !" 7 ) from the center of the host galaxy.

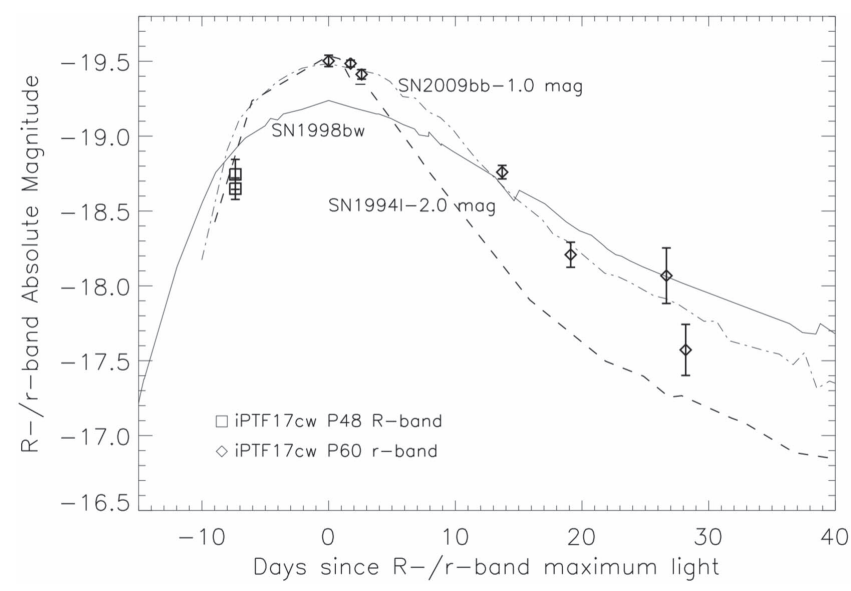

Figure 3. $P 48 R$-band and $P 60 r$-band light curves of iPTF17cw (corrected for galactic extinction) compared to the (extinction-corrected) $R$-band light curves of: the GRB-associated BL-Ic SN 1998bw (solid; Clocchiatti et al. 2011); the relativistic BL-Ic SN 2009bb (dashed-dotted; Pignata et al. 2011); type Ic SN 1994I (dashed; Richmond et al. 1996). Both the SN 2009bb and SN 1994I light curves have been scaled to match the peak luminosity of iPTF17cw.

Masci et al. 2017). iPTF17cw is located at $\alpha=09^{\mathrm{h}} 03^{\mathrm{m}} 38^{\mathrm{s}} .38$ and $\delta=+43^{\circ} 05^{\prime} 50$ ". 3 (J2000; Figure 2), at an angular separation of $\approx 1$ !' 7 from the nominal position of SDSS J090338.47+430551.6 (York et al. 2000).

At the time of discovery, iPTF17cw was visible at a magnitude of $R \approx 19.5 \mathrm{mag}$. The $\mathrm{SN}$ was not visible in a previous $g$-band image of the field obtained on 2016 December 6 with the P48. Subsequent observations were performed with the Palomar 60 inch Telescope (P60; Cenko et al. 2006) in gri bands. Photometry was performed relative to the Sloan Digital Sky Survey (SDSS) $r$ - and $g$-band magnitudes of stars in the field, using our custom pipelines (Ofek et al. 2012; Fremling et al. 2016).

In Figure 3 we show the P48 $R$-band and P60 $r$-band light curves of iPTF17cw. In Table 1 we report the multi-color photometry of iPTF17cw. 
Table 1

Optical Photometry of iPTF17cw

\begin{tabular}{|c|c|c|c|}
\hline $\begin{array}{l}\text { Date } \\
\text { (MJD) }\end{array}$ & $\begin{array}{l}\text { Tele- } \\
\text { scope }\end{array}$ & Band & $\begin{array}{l}\text { Magnitude } \\
\text { [AB] }\end{array}$ \\
\hline 57760.291 & $\mathrm{P} 48$ & $R$ & $19.46 \pm 0.10$ \\
\hline 57760.319 & P48 & $R$ & $19.557 \pm 0.072$ \\
\hline 57768.305 & P60 & $g$ & $19.041 \pm 0.077$ \\
\hline 57770.194 & P60 & $g$ & $19.235 \pm 0.034$ \\
\hline 57771.149 & P60 & $g$ & $19.294 \pm 0.054$ \\
\hline 57780.219 & P60 & $g$ & $20.54 \pm 0.17$ \\
\hline 57783.277 & P60 & $g$ & $>20.6$ \\
\hline 57787.103 & P60 & $g$ & $>17.6$ \\
\hline 57789.186 & P60 & $g$ & $20.93 \pm 0.16$ \\
\hline 57793.187 & P60 & $g$ & $>19.8$ \\
\hline 57768.301 & P60 & $r$ & $18.703 \pm 0.038$ \\
\hline 57770.191 & P60 & $r$ & $18.721 \pm 0.022$ \\
\hline 57771.146 & P60 & $r$ & $18.793 \pm 0.032$ \\
\hline 57783.273 & P60 & $r$ & $19.446 \pm 0.045$ \\
\hline 57784.124 & P60 & $r$ & $>18.4$ \\
\hline 57789.183 & P60 & $r$ & $19.998 \pm 0.084$ \\
\hline 57793.184 & P60 & $r$ & $>18.3$ \\
\hline 57794.123 & P60 & $r$ & $>19.4$ \\
\hline 57797.444 & P60 & $r$ & $20.14 \pm 0.19$ \\
\hline 57799.108 & P60 & $r$ & $20.63 \pm 0.17$ \\
\hline 57768.303 & P60 & $i$ & $18.901 \pm 0.066$ \\
\hline 57770.193 & P60 & $i$ & $18.872 \pm 0.040$ \\
\hline 57771.147 & P60 & $i$ & $18.902 \pm 0.053$ \\
\hline 57780.217 & P60 & $i$ & $19.142 \pm 0.060$ \\
\hline 57783.275 & P60 & $i$ & $19.223 \pm 0.067$ \\
\hline 57789.185 & P60 & $i$ & $19.692 \pm 0.062$ \\
\hline 57793.185 & P60 & $i$ & $>19.0$ \\
\hline 57794.125 & P60 & $i$ & $>18.1$ \\
\hline 57797.446 & P60 & $i$ & $20.17 \pm 0.17$ \\
\hline 57799.110 & P60 & $i$ & $>20.3$ \\
\hline
\end{tabular}

\subsection{Spectral Classification}

A summary of our spectroscopic observations of iPTF17cw is reported in Table 2.

We observed iPTF17cw with the Double Spectrograph (DBSP; Oke \& Gunn 1982) on the Palomar $5.1 \mathrm{~m}$ Hale telescope (P200) for 600 s beginning at 13:20 UT on 2017 January 7 (MJD 57760.555; Figure 4, blue). The blue channel used the 600 lines/mm grating, giving an effective wavelength coverage of 3085-5840 $\AA$. The red channel used the 316 lines/ mm grating, which covers the range 5200-10900 $\mathrm{A}$. The D55 dichroic was used to divide light into the two channels $(50 \%$ transmission at $5500 \AA$ ). The observations used a $1^{\prime \prime}$ slit mask oriented close to the parallactic angle (Filippenko 1982). The DBSP data were processed and optimally extracted using a custom pipeline implemented in IRAF, ${ }^{14}$ python, and IDL. Background light was fit for and removed from the 2D frames using the IDL routine bspline_itterfit.pro following the procedure described by Kelson (2003). An initial wavelength solution was found using calibration lamp observations. This solution was then adjusted based on night sky lines to account for flexture in the instrument. The flux

\footnotetext{
14 IRAF is distributed by the National Optical Astronomy Observatory, which is operated by the Association of Universities for Research in Astronomy (AURA) under a cooperative agreement with the National Science Foundation.
}

scale was determined using observations of the standard star Feige34 taken on the same night.

Narrow emission lines are detected at $4073 \AA, 5471 \AA$, and $7171 \AA$. Identifying these as O II $\lambda \lambda 3726,3728$, O III 5007, and $\mathrm{H} \alpha$ from the host galaxy, we determine a redshift of $z=0.093$. Aside from these narrow features, the spectra show subtle, broad features superposed on a continuum that peaks around $3800 \AA$ in the rest frame (Figure 4, left, blue line). Using the spectral template fitting code, superfit (Howell et al. 2006), we find that the spectrum is reasonably well matched to the BL-Ic SN 1998bw around maximum light (Figure 4, right; see also Iwamoto et al. 1998).

An additional spectrum of iPTF17cw was obtained by combining three spectra collected around a similar epoch (2017 January 9 UT) using the SPRAT spectrograph (Piascik et al. 2014) on the $2 \mathrm{~m}$ robotic Liverpool Telescope. SPRAT is a long-slit spectrograph with a wavelength range of $4000-8000 \AA$ and a resolving power of $R \sim 350$ at the center of this range. These data were reduced, wavelength-calibrated, and flux-calibrated using the automated Liverpool Telescope spectroscopic pipeline, and the combined spectrum was obtained using STARLINK/FIGARO software (Currie et al. 2014). The SPRAT spectrum of iPTF17cw is consistent with the above described P200 spectrum collected at a similar epoch (see Figure 5).

We continued monitoring of iPTF $17 \mathrm{cw}$ with the DeVeny spectrograph mounted on the $4.3 \mathrm{~m}$ Discovery Channel Telescope (DCT) in Happy Jack, Arizona. We obtained a series of $4 \times 600 \mathrm{~s}$ exposures beginning at 8:36 UT on 1 February 2017 (MJD 57785.358). We employed the 300 lines $\mathrm{mm}^{-1}$ grating with a $1.5 \AA$ slit, resulting in a spectral resolution of $\approx 10 \AA$ and wavelength coverage from $\approx 3500$ to $8000 \AA$. All spectra were obtained at the parallactic angle (Filippenko 1982) and reduced in the IRAF environment using standard routines. Spectra were extracted optimally (Horne 1986), and we performed wavelength calibration first relative to $\mathrm{HgNeArCd}$ arc lamps and then tweaked based on night sky lines. Extracted spectra were divided through by a smoothed flux standard to remove narrowband $(<50 \AA)$ instrumental effects (Bessell 1999). Finally, telluric atmospheric absorption features were removed using the continuum from spectrophotometric standards (Wade \& Horne 1988). The resulting spectrum is shown in Figure 4 (green).

A last spectrum of iPTF17cw was taken with the Keck I/ LRIS instrument (Oke et al. 1995) at 10:53:39 UT on 2017 February 27 (MJD 57811.454). The instrument was configured in long-slit mode, with a $1^{\prime \prime}$ slit and 400/3400 + 400/8500 grism+grating combination (dispersion of 1.09 and $1.16 \AA$ / pixel, respectively). The total exposure time was $1800 \mathrm{~s}$ in the blue channel and $1700 \mathrm{~s}$ in the red. The resolution of the spectrum, as measured from the O I $5577 \AA$ line, is of $9.6 \AA$ $\left(\approx 440 \mathrm{~km} \mathrm{~s}^{-1}\right)$. The data were reduced using a custom developed pipeline in IDL, LPIPE. The standard stars Feige34 and Feige67 were used for flux calibration and correction for telluric lines. This spectrum is show in Figure 4 (red).

\subsection{Radio Follow-up}

Radio observations of the iPTF17cw field were carried out with the Karl G. Jansky Very Large Array ${ }^{15}$ (VLA) in its A,

\footnotetext{
15 The National Radio Astronomy Observatory is a facility of the National Science Foundation operated under cooperative agreement by Associated Universities, Inc.
} 

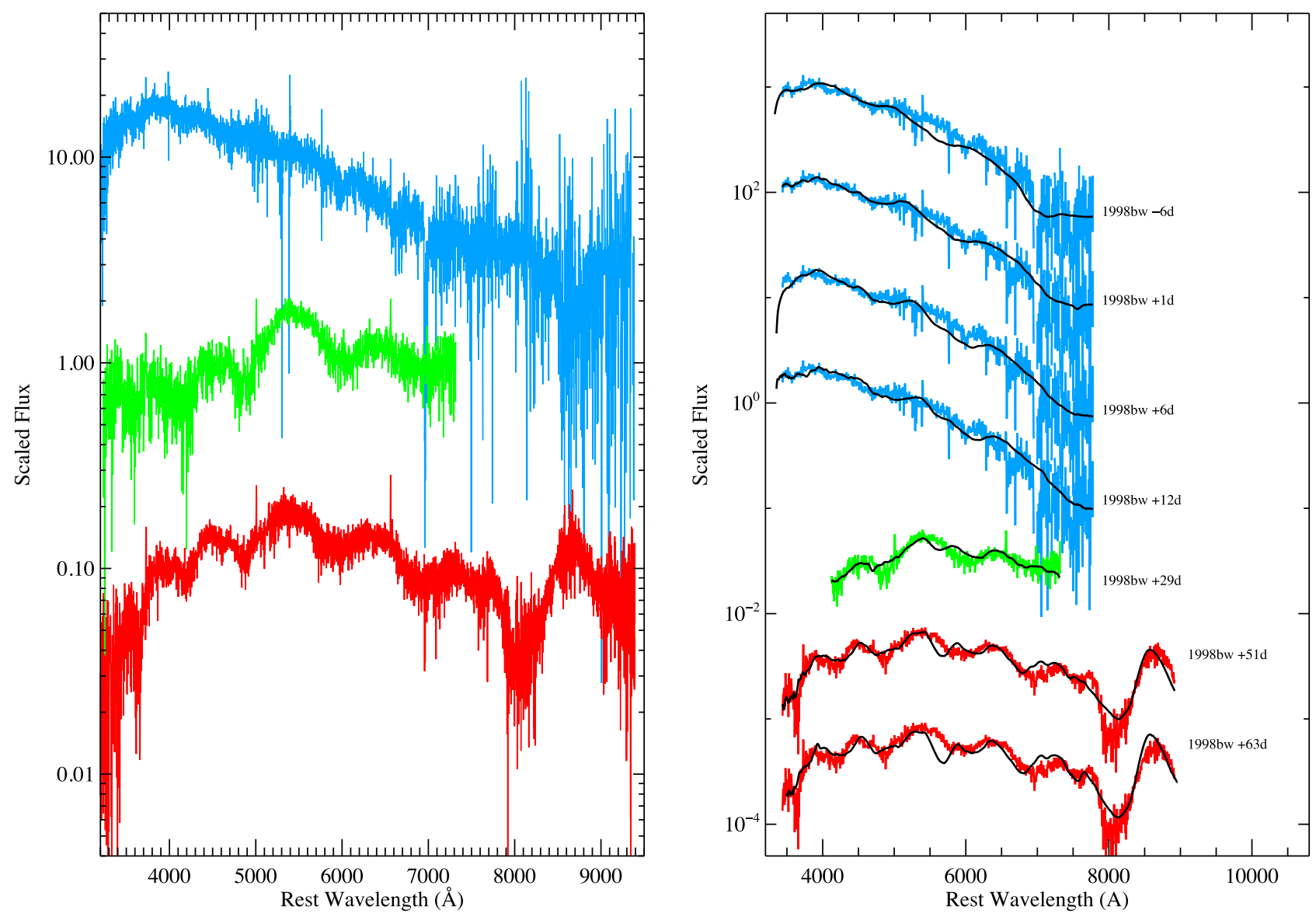

Figure 4. Left: iPTF17cw spectra (blue for P200/DBSP, $\approx-8$ days since $r$-band maximum light; green for DCT, $\approx+17$ days since $r$-band maximum light; red for Keck/LRIS, $\approx+43$ days since $r$-band maximum light), corrected for redshift effects assuming $z=0.093$. Right: same iPTF spectra as in the left panel, but binned and galaxy-template-subtracted, compared to de-reddened SN 1998bw spectra at various phases (see the text for a discussion).

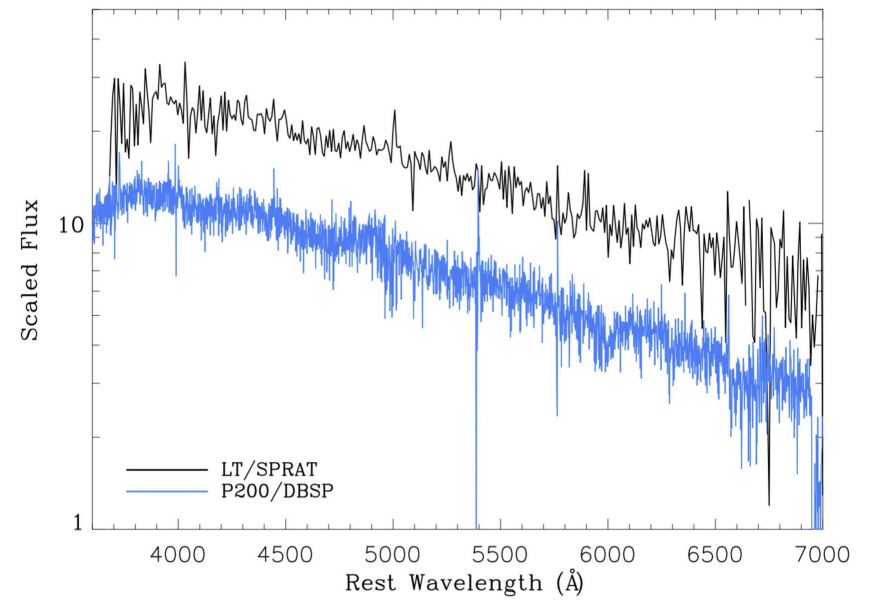

Figure 5. LT/SPRAT spectrum of iPTF17cw (at $\approx-6$ days since $r$-band maximum light) compared to the P200/DBSP spectrum (at $\approx-8$ days since $r$-band maximum light).

$\mathrm{AnD}$, and $\mathrm{D}$ configurations, under our target of opportunity program. ${ }^{16}$ Our first observation was carried out in the C-band (nominal central frequency of $\approx 6 \mathrm{GHz}$ ) on 2017 January 13 (approximately 6 days since P48 discovery). VLA follow-up observations were continued for up to about three months since optical discovery. We used $\mathrm{J} 0920+4441$ as our phase

16 VLA/16B-043; PI: A. Corsi. calibrator, and 3C48 or 3C286 as flux and bandpass calibrators. VLA data were reduced and calibrated using the VLA automated calibration pipeline, which runs in the Common Astronomy Software Applications package (CASA; McMullin et al. 2007). When necessary, additional flags were applied after visual inspection of the data. Images of the observed field were formed using the CLEAN algorithm (Högbom 1974), which we ran in interactive mode. The results of our VLA follow-up campaign of iPTF17 cw are reported in Table 3. Flux measurement errors are calculated as the quadratic sum of the map rms, plus a 5\% fractional error on the measured flux, which accounts for the inaccuracies of the flux density calibration.

A faint radio source was detected at a location compatible with the optical position of iPTF17cw, at a $\approx 5 \sigma$ level during our first observation at $\approx 6 \mathrm{GHz}$. The source appears to be point-like, with the VLA in its A configuration (nominal FWHP at $6 \mathrm{GHz}$ of $\approx 0$ !" 33), thus a contribution from star formation in the underlying host galaxy is unlikely. The positional offset with respect to the reddish central concentration of the iPTF17 $\mathrm{cw}$ host galaxy (Figure 2) also makes a contribution from a low-level AGN unlikely. In Figure 6 we show the VLA images of the iPTF $17 \mathrm{cw}$ radio counterpart collected during our first multi-band observation of this source.

Over the first three C-band epochs, which cover a time interval of $\approx 10$ days, the source radio flux at $\approx 6 \mathrm{GHz}$ shows marginal evidence $(\approx 2 \sigma$ level) for variability, suggesting a decay of the radio flux with time $\left(f_{6 \mathrm{GHz}} \propto\left(t-t_{0}\right)^{\alpha}\right.$ with 
Table 2

Spectroscopic Observations of iPTF17cw

\begin{tabular}{lclcr}
\hline \hline $\begin{array}{l}\text { Date } \\
(\mathrm{MJD})\end{array}$ & $\begin{array}{c}\text { Phase } \\
(\text { day })\end{array}$ & Instrument & $\begin{array}{c}\text { Wavelength Range } \\
(\mathrm{A})\end{array}$ & $\begin{array}{r}\text { Exp. Time } \\
(\mathrm{s})\end{array}$ \\
\hline 57760.555 & $\approx-8$ & P200/DBSP & $3085-10900$ & 600 \\
55762.249 & $\approx-6$ & LT/SPRAT & $4000-8000$ & $3 \times 600$ \\
57785.358 & $\approx+17$ & DCT/DeVeny & $3500-8000$ & 600 \\
57811.454 & $\approx+43$ & Keck I/LRIS & $3150-9400$ & $1800 / 1700$
\end{tabular}

Note. Phases are in days since observed $g$-band and $r$-band maximum light. All spectra will be made available via WISeREP (Yaron \& Gal-Yam 2012).

Table 3

Radio Observations of iPTF17cw

\begin{tabular}{|c|c|c|c|c|}
\hline MJD & $\begin{array}{c}\text { Epoch } \\
\text { (day) }\end{array}$ & Instr.:config & $\begin{array}{l}\text { Freq. } \\
(\mathrm{GHz})\end{array}$ & $\begin{array}{l}\text { Flux Density } \\
\left(\mu \mathrm{Jy} \text { beam }^{-1}\right)\end{array}$ \\
\hline 57763.140 & 12.6 & VLA:A & 6.2 & $38.1 \pm 7.3$ \\
\hline 57766.260 & 15.7 & VLA:A & 6.3 & $30.4 \pm 5.9$ \\
\hline $\begin{array}{l}57766.464 \\
,\end{array}$ & $\begin{array}{l}15.9 \\
"\end{array}$ & $\begin{array}{l}\text { VLA:A } \\
\text { " }\end{array}$ & $\begin{array}{l}2.8 \\
14\end{array}$ & $\begin{aligned} 50 & \pm 10 \\
25.4 & \pm 6.2\end{aligned}$ \\
\hline 57772.115 & 21.6 & VLA:A & 6.2 & $19.9 \pm 6.2$ \\
\hline 57775.451 & 24.9 & $\begin{array}{c}\text { VLA:A } \\
, "\end{array}$ & $\begin{array}{l}2.8 \\
14\end{array}$ & $\begin{array}{l}41.4 \pm 8.7 \\
21.1 \pm 6.3\end{array}$ \\
\hline 57781.217 & 30.7 & VLA:AnD & 6.2 & $22.4 \pm 5.5$ \\
\hline $\begin{array}{l}57792.182 \\
\text { " }\end{array}$ & 41.6 & $\begin{array}{c}\text { VLA:AnD } \\
\quad "\end{array}$ & $\begin{array}{l}2.8 \\
6.2\end{array}$ & $\begin{array}{l}44 \pm 14 \\
19 \pm 20\end{array}$ \\
\hline 57816.103 & 65.6 & VLA:D & 14 & $20.2 \pm 6.8$ \\
\hline 57855.470 & 105 & VLA:D & 14 & $10.7 \pm 5.2$ \\
\hline
\end{tabular}

Note. Epochs are calculated since the Fermi/GBM trigger time of GRB 161228B (MJD 57750.552).

$\alpha=-1.18 \pm 0.68$ when $t_{0}$ is set equal to the trigger time of GRB 161228B; see Section 2.5), perhaps followed by what appears to be a flattening or re-brightening of the $6 \mathrm{GHz}$ emission (Figure 7, top). Due to the change of the VLA configuration and to the presence of a bright QSO in the field, our latest VLA image of the iPTF17cw field at $6 \mathrm{GHz}$ is substantially degraded (the image is dynamic-range-limited) and resulted in a non-detection.

The candidate radio counterpart to $\mathrm{iPTF} 17 \mathrm{cw}$ was also detected at the $\gtrsim 3 \sigma$ level at 2.8 and $14 \mathrm{GHz}$. The observations at such frequencies do not show evidence for substantial variability over the first two months of observations, but our last observation at $14 \mathrm{GHz}$ shows that the radio counterpart to iPTF17cw has faded below our detection level.

\subsection{X-Ray Observations}

The Swift satellite (Gehrels et al. 2005) observed the location of iPTF17cw on two epochs, beginning at 13:48 UT on 2017 January 13, and 00:35 UT on 2017 January 18. These observations were obtained as part of our approved target of opportunity program (Table 4). ${ }^{17}$ We analyzed the data obtained by the onboard X-Ray

${ }^{17}$ Swift Cycle 12 GI; PI: A. Corsi.
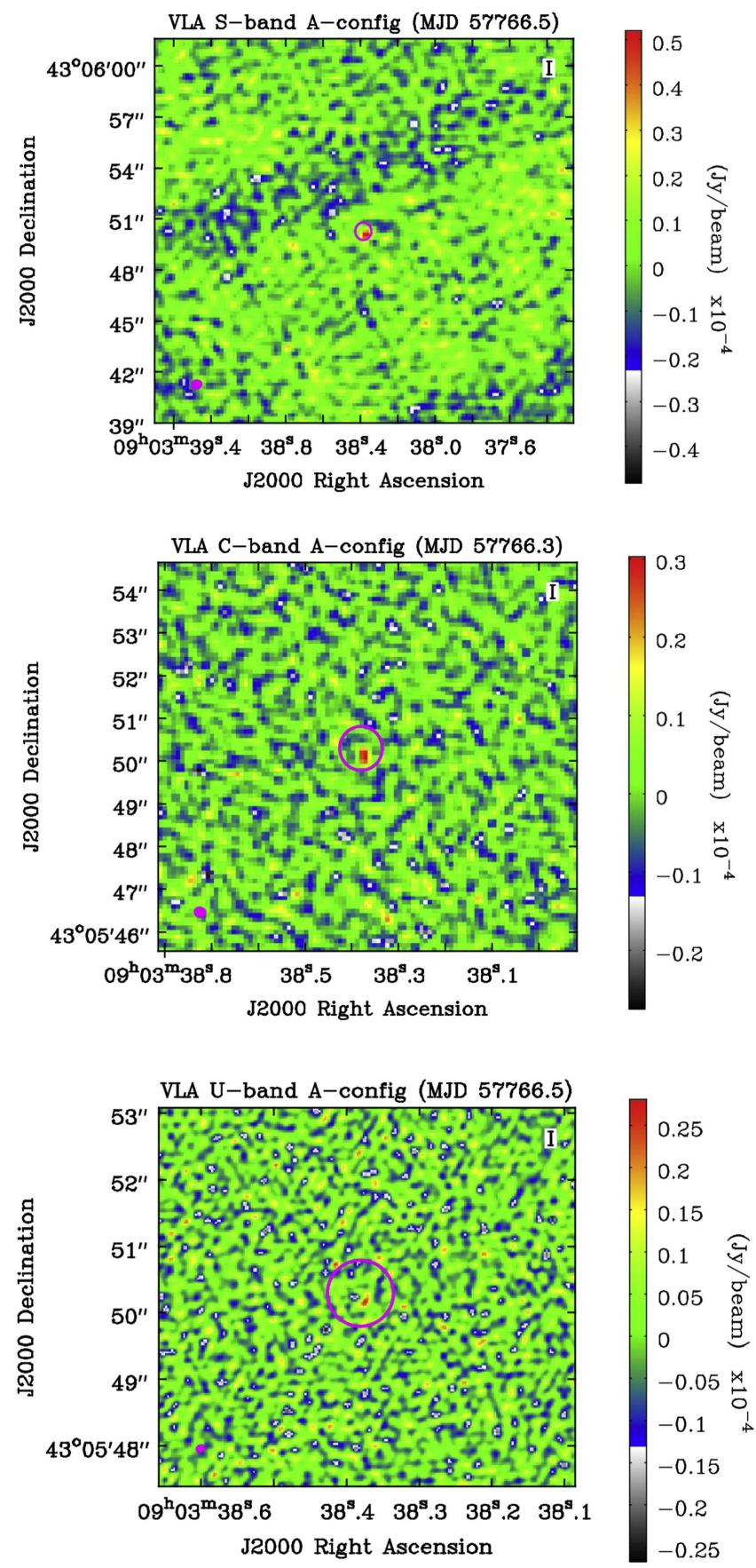

Figure 6. Radio images ( $2.8 \mathrm{GHz}$, top; $6 \mathrm{GHz}$, middle; $14 \mathrm{GHz}$, bottom) of the iPTF $17 \mathrm{cw}$ field as seen by the VLA in its A configuration. The optical position of iPTF $17 \mathrm{cw}$ is at the center of the 0"5-radius magenta circle (iPTF localization). The size of the VLA beam at each frequency is shown as a magenta filled ellipse in the bottom left corner of each panel. The radio counterpart of iPTF17cw is within the iPTF localization circle and appears to be point-like at all frequencies (as evidenced by comparing its size with the size of the synthesized VLA beam in magenta).

Telescope (XRT; Burrows et al. 2005) during these two epochs $(1.4$ and $7.5 \mathrm{ks}$ of exposure time, respectively) using the procedures outlined in Evans et al. (2009). No X-ray emission was detected at the location of iPTF17cw. We place $(3 \sigma)$ upper limits on the $0.3-10 \mathrm{keV}$ count rate of $<8.1 \times 10^{-3}$ and $<2.0 \times 10^{-3}$ count s$^{-1}$ in these two epochs. For a power-law spectrum with a photon index of $\Gamma=2$ and a Galactic $\mathrm{H}$ I column density of $N_{H} \approx 1.8 \times 10^{20} \mathrm{~cm}^{-2}$ (Dickey \& Lockman 1990), 

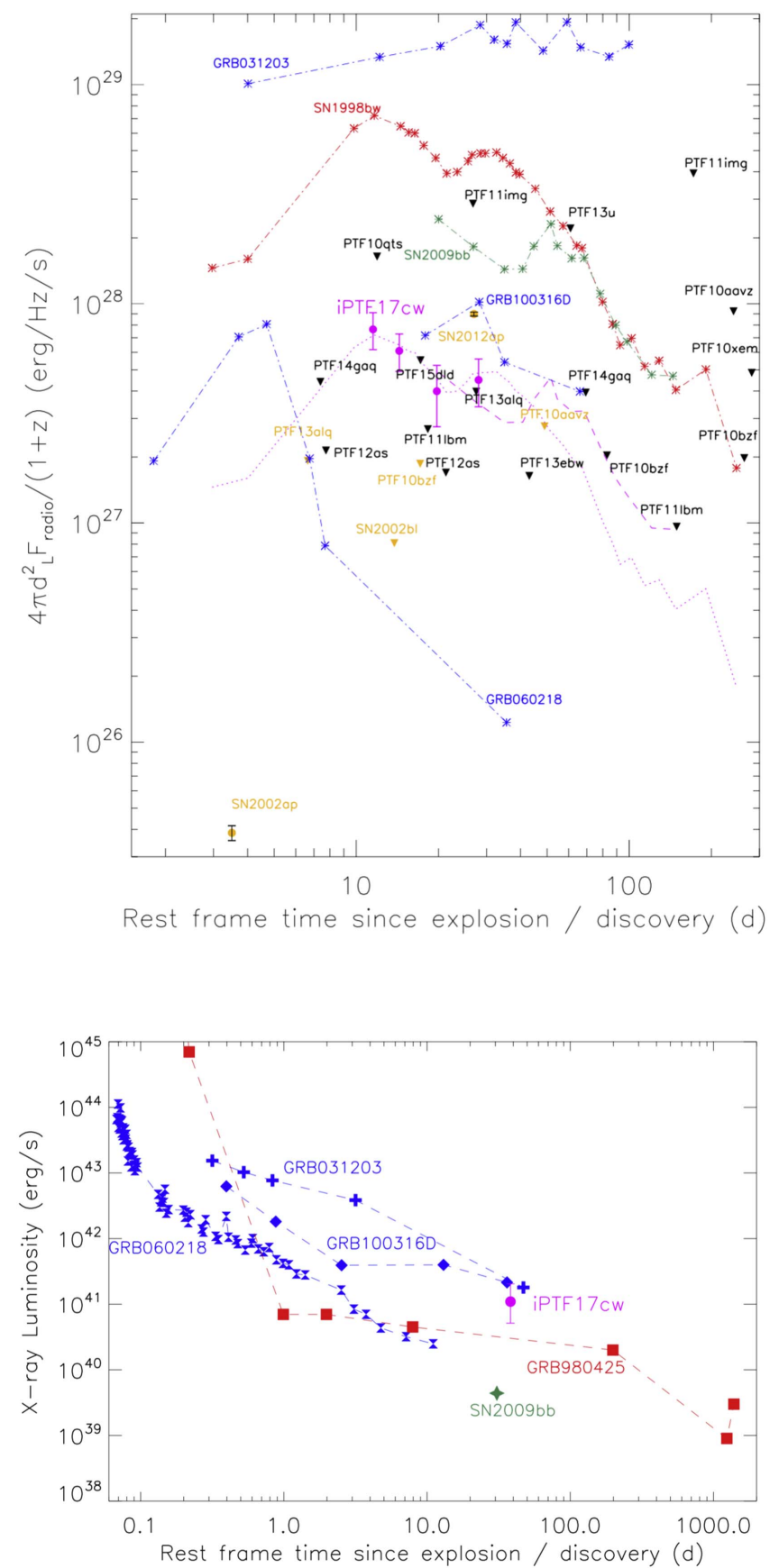

Figure 7. Top: radio $(\approx 5 \mathrm{GHz})$ light curves of some low-luminosity GRBs (blue; Soderberg et al. 2004, 2006b; Margutti et al. 2013), of the GRB-associated BL-Ic SN 1998bw (red; Kulkarni et al. 1998), the relativistic BL-Ic SN 2009bb (green; Soderberg et al. 2010), and SN 2012ap (yellow dot; Chakraborti et al. 2015), and the non-relativistic BL-Ic SN 2002ap (yellow dot; Berger et al. 2003a), are compared to the $6 \mathrm{GHz}$ radio emission from iPTF17cw (magenta dots). The light curves of SN 1998bw and SN 2009bb, scaled to match the radio luminosity of iPTF17cw, are also shown for comparison (magenta dotted and dashed lines, respectively). We also show upper limits for the sample of BL-Ic SNe without a radio detection collected as part of our (black downward pointing triangles) or other (yellow downward pointing triangles) programs. Here we do not show BL-Ic SNe in our sample with radio detections due to strong CSM interaction (Corsi et al. 2014, 2016). See the text for discussion. Bottom: X-ray luminosity of some of the low-luminosity GRBs (Kouveliotou et al. 2004; Watson et al. 2004; Campana et al. 2006; Margutti et al. 2013) and relativistic SNe (Soderberg et al. 2010) plotted in the top panel, compared to iPTF17cw. See the text for discussion.

these correspond to $(3 \sigma)$ limits on the $0.3-10 \mathrm{keV}$ (unabsorbed) $\mathrm{X}$-ray flux of $<3.4 \times 10^{-13}$ and $<8.5 \times 10^{-14} \mathrm{erg} \mathrm{cm}^{-2} \mathrm{~s}^{-1}$, respectively.

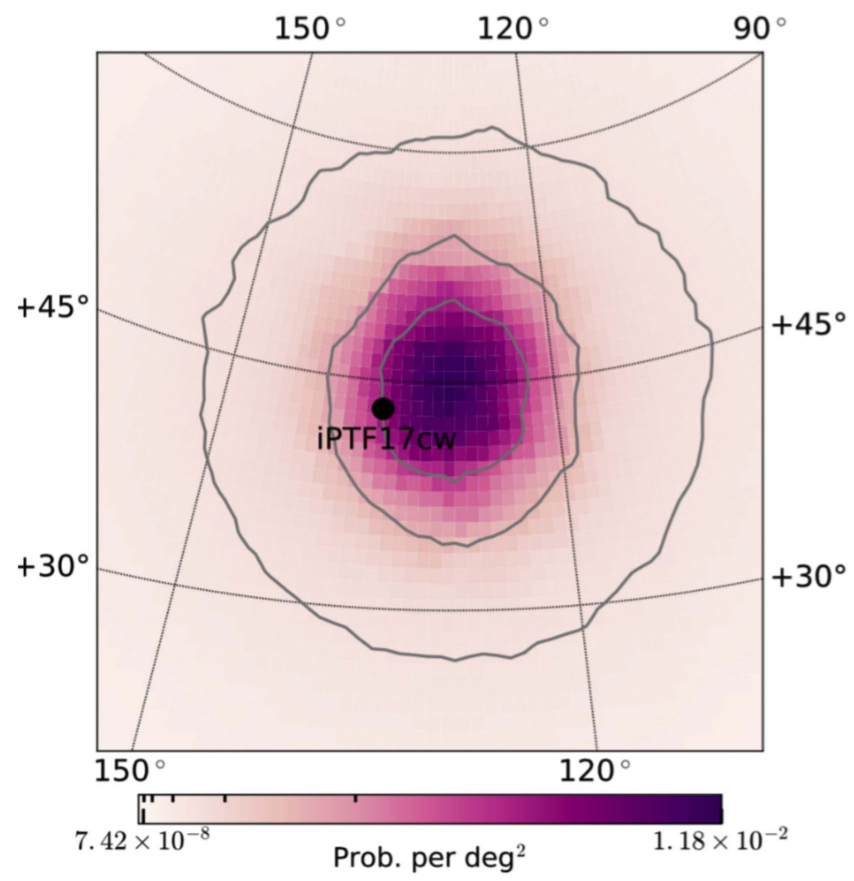

Figure 8. GBM localization for GRB 161228B. The three gray contours denote the $1 \sigma, 2 \sigma$, and $3 \sigma$ confidence regions, and the purple color gradient shows the probability per square degree for the true location of the source. The location of iPTF17cw is shown to exist near the $1 \sigma$ confidence contour.

We also observed the location of iPTF17cw with the Advanced CCD Imaging Spectrometer (ACIS) on the Chandra X-ray Observatory ${ }^{18}$ beginning at 9:22 UT on 2017 February 8. The source location was positioned on the S3 chip and the detector data was telemetered in "faint" mode. An effective exposure time of $9.86 \mathrm{ks}$ was obtained on source. We detect a total of 5 photons within $1^{\prime \prime}$ (radius) of the position of iPTF17cw. While not sufficient for any spectral analysis, we can claim a detection at the $\approx 3 \sigma$ confidence level (expected number of background counts: 0.89 ).

The position of the iPTF17cw X-ray counterpart as seen by Chandra is $\alpha=09^{\mathrm{h}} 03^{\mathrm{m}} 38.37$ and $\delta=+43^{\circ} 05^{\prime} 50^{\prime \prime} 4(\mathrm{~J} 2000)$, with an estimated (90\% confidence) error radius of $\approx 0$ ". 6 (dominated by Chandra pointing errors). This position is compatible with both the optical position of iPTF17cw, and the position of its radio counterpart (see Figure 2).

Properly accounting for the on-axis point-spread function we measure a $0.5-7.0 \mathrm{keV}$ count rate of $5.0_{-2.9}^{+4.8} \times 10^{-4}$ count s$^{-1}$ (90\% confidence interval). Adopting a power-law spectral model with photon index $\Gamma=2$ and $N_{H} \approx 1.8 \times 10^{20} \mathrm{~cm}^{-2}$ (Dickey \& Lockman 1990), this corresponds to an unabsorbed X-ray flux in this bandpass of $f_{X}=\left(5.0_{-2.9}^{+4.7}\right) \times 10^{-15} \mathrm{erg} \mathrm{cm}^{-2} \mathrm{~s}^{-1}$, or an $\mathrm{X}$-ray luminosity of $\approx(4-19) \times 10^{40} \mathrm{erg} \mathrm{s}^{-1}$ (Figure 7 , bottom).

\subsection{Search for $\gamma$-rays}

We searched the Fermi and Swift catalogs for potential GRBs detected within a month prior to the iPTF discovery of iPTF17cw, and with a position compatible with that of iPTF $17 \mathrm{cw}$. We found a match with GRB 161228B (Figure 8), which was observed by Fermi/GBM as trigger \#504623745 on ${ }^{19} 2016$ December 28, at

\footnotetext{
18 Program ID: \#17508570; PI: Corsi.

19 https://gcn.gsfc.nasa.gov/other/504623745.fermi
} 
13:15:41.85 UT (MJD 57750.552). This GRB was also detected by POLAR and Integral (Marcinkowski et al. 2016).

GRB 161228B, as observed by Fermi/GBM, appears to be approximately $30 \mathrm{~s}$ in duration. Utilizing the position of iPTF17cw for the GBM detector responses, we performed a time-integrated spectral analysis starting at about $1 \mathrm{~s}$ before the GBM trigger time and extending to $30 \mathrm{~s}$ after trigger time. The data are well-fit by a Band function (defined as in Gruber et al. 2014) with the following parameter values: $E_{\text {peak }}=195 \pm 47 \mathrm{keV}$; low-energy power-law index, $\alpha=-1.2 \pm 0.1 ;$ and high-energy power-law index, $\beta=-2.3 \pm 0.5$. The derived fluence from this spectral fit in the typical GBM reporting band of $10-1000 \mathrm{keV}$ is $(5.6 \pm 0.4) \times$ $10^{-6} \mathrm{erg} \mathrm{cm}^{-2}$. Additionally, we analyzed the brightest $1 \mathrm{~s}$ interval of data for this GRB to estimate the peak flux. The high-energy power-law index of the Band function could not be adequately constrained for this interval, however, a power-law with an exponential cutoff could be reasonably constrained with parameter values of $E_{\text {peak }}=214 \pm 26 \mathrm{keV}$ and power-law index $\alpha=$ $0.3 \pm 0.3$. The $1 \mathrm{~s}$ peak flux is then estimated to be $(5.4 \pm 0.9) \times 10^{-7} \mathrm{erg} \mathrm{s}^{-1} \mathrm{~cm}^{-2}$.

\section{Multi-wavelength Analysis}

\subsection{Photometric Properties}

To compare the intrinsic peak luminosity of iPTF $17 \mathrm{cw}$ with that of other GRB-associated SNe, we use our gri photometry from P60 and the prescriptions of Lyman et al. (2014) for transforming optical light curves of stripped-envelope corecollapse SNe to full bolometric light curves. We define the full bolometric correction in the $g$-band as (Lyman et al. 2014)

$$
\mathrm{BC}_{g, \text { peak }}=M_{\text {bol,peak }}-M_{g, \text { peak }} \text {, }
$$

where

$$
M_{\mathrm{bol}, \text { peak }}=M_{\odot, \text { bol }}-2.5 \log _{10}\left(\frac{L_{\text {bol, peak }}}{L_{\odot, \text { bol }}}\right) .
$$

In the above relation, $M_{\odot, \text { bol }}=4.74$ mag and $L_{\odot, \text { bol }}=3.828 \times$ $10^{33} \mathrm{erg} \mathrm{s}^{-1}$.

Following Lyman et al. (2014), we express $\mathrm{BC}_{g}$ as a function of $g-i$ or $g-r$ colors. For stripped-envelope core-collapse $\mathrm{SNe}$, the best-fit relations between colors and bolometric corrections give

$$
\begin{gathered}
\mathrm{BC}_{g}=0.054-0.195 \times(g-r)-0.719 \times(g-r)^{2}, \\
\mathrm{BC}_{g}=-0.029-0.404 \times(g-i)-0.230 \times(g-i)^{2} .
\end{gathered}
$$

To derive the iPTF $17 \mathrm{cw}$ colors, we correct our photometry for Galactic extinction toward the position of iPTF17cw $\left(A_{V}=0.0517 \mathrm{mag}\right.$, which, assuming an extinction to reddening ratio $A_{V} / E(B-V)=3.1$, implies $A_{g}=0.064 \mathrm{mag}, A_{r}=$ $0.044 \mathrm{mag}, A_{i}=0.033 \mathrm{mag}$; Schlafly \& Finkbeiner 2011). This yields gri-band extinction-corrected magnitudes of iPTF17cw around the time of the $r$-band peak (MJD $\approx 57768$; see Figure 3 and Table 1) of $g=18.977 \pm 0.077 \mathrm{mag}, \quad r=18.665 \pm$ $0.038 \mathrm{mag}$, and $i=18.868 \pm 0.066 \mathrm{mag}$, respectively (see Table 1). Inserting these into Equations (3)-(4), we get two consistent values of the bolometric correction at MJD $\approx 57768$, $\mathrm{BC}_{g} \approx-0.077 \mathrm{mag}$ and $\mathrm{BC}_{g} \approx-0.076 \mathrm{mag}$, respectively. We stress that these are tentative estimates of the bolometric correction based on the results of Lyman et al. (2014) from best-fit relations between colors and bolometric corrections for a population of stripped-envelope core-collapse $\mathrm{SNe}$. As discussed in Lyman et al. (2014), the population scatter around the best-fit relations is $\approx 0.076 \mathrm{mag}$ for the $g$-band bolometric correction based on the $g-r$ color, and 0.060 mag for the one based on the $g-i$ color. We thus set $\mathrm{BC}_{g}=(-0.077 \pm 0.069)$ mag.

From Equation (1) we thus derive $M_{\text {bol }} \lesssim-19.26 \pm$ 0.10 mag (where the upper limit reflects the fact that we do not have $g$-band photometry available before MJD $\approx 57768$, but we know that the $g$-band light curve decays after this time; see Table 1), which implies $L_{\text {bol,peak }} \gtrsim 10^{43} \mathrm{erg} \mathrm{s}^{-1}$. This is comparable to the bolometric luminosity of the engine-driven SN 1998bw, for which $L_{\text {bol,peak }} \approx(7.7 \pm 8.6) \times 10^{42} \mathrm{erg} \mathrm{s}^{-1}$ (see Table 3 in Clocchiatti et al. 2011). Applying k-corrections and host extinction corrections based on colors, we find that iPTF $17 \mathrm{cw}$ is, in terms of optical brightness, among the top $\approx 10 \%$ of the iPTF BL-Ic SN sample (F. Taddia et al. 2017, in preparation). From the bolometric peak luminosity we can estimate the amount of ${ }^{56} \mathrm{Ni}$ in the explosion, via the relation (e.g., Arnett 1982; Lyman et al. 2016)

$$
\log _{10}\left(M_{\mathrm{Ni}} / M_{\odot}\right)=-0.415 \times M_{\text {bol,peak }}-8.184,
$$

which yields $M_{\mathrm{Ni}} \approx 0.644_{-0.059}^{+0.065} M_{\odot}$ for iPTF17cw, comparable to the literature values range of $(0.4-0.7) M_{\odot}$ for SN 1998bw, and somewhat higher than the $M_{\mathrm{Ni}} \approx 0.25 M_{\odot}$ estimated for SN 2009bb (see Table 5 in Lyman et al. 2016).

As evident from Figure 3, the $r$-band light curve shape of iPTF17cw is more similar to that of SN 2009bb (Pignata et al. 2011) than to that of SN 1998bw. The light curve shape is related to the characteristic timescale scale (e.g., Arnett 1982; Lyman et al. 2016):

$$
\tau_{m} \approx 7.7 \text { days }\left(\frac{M_{\text {eje }}}{M_{\odot}}\right)^{3 / 4}\left(\frac{E_{K}}{10^{51} \mathrm{erg}}\right)^{-1 / 4},
$$

where $M_{\text {eje }}$ is the ejecta mass, $E_{K}$ is the kinetic energy, and where we have assumed an opacity of $\approx 0.06 \mathrm{~cm}^{2} \mathrm{~g}^{-1}$. For $\mathrm{SN} 2009 \mathrm{bb}, \tau_{m, \mathrm{bb}} \approx 10$ days (Lyman et al. 2016), and given the similarity with the light curve of iPTF17cw, we derive

$$
\left(\frac{E_{K}}{10^{51} \mathrm{erg}}\right) \approx 0.35 \times\left(\frac{M_{\mathrm{eje}}}{M_{\odot}}\right)^{3} .
$$

\subsection{Spectroscopic Properties}

In Figure 4 (right) we show the DBSP spectrum of iPTF17cw (see Section 2.1) collected on MJD 57760.555 (at a phase of $\approx-8$ days since $r$-band maximum light), compared to SN 1998bw spectra at several phases since maximum light. The SN 1998bw spectra shown in this Figure have been "dereddened" significantly to match the iPTF $17 \mathrm{cw}$ spectrum and to account for the much bluer continuum of iPTF $17 \mathrm{cw}$ at early times. The iPTF17cw DBSP spectrum was corrected for host galaxy contribution (a host galaxy light template was estimated using Superfit and then subtracted to the iPTF17cw spectrum). A comparison of the spectra shown in the left and right panels of Figure 4 gives an idea of the effects of these corrections.

In Figure 4 (right) we also show the DCT and Keck/LRIS spectra of iPTF17cw taken on MJD $57785.358(\approx+17$ days 
Table 4

X-Ray Observations of iPTF17cw

\begin{tabular}{|c|c|c|c|c|c|c|}
\hline $\begin{array}{l}\text { Date } \\
\text { (MJD) }\end{array}$ & $\begin{array}{l}\text { Epoch } \\
\text { (day) }\end{array}$ & Instrument & $\begin{array}{l}\text { Band } \\
(\mathrm{keV})\end{array}$ & $\begin{array}{c}\text { Exp. Time } \\
(\mathrm{ks})\end{array}$ & $\begin{array}{l}\text { Count Rate } \\
\qquad\left(s^{-1}\right)\end{array}$ & $\begin{array}{l}\text { Flux (unabs) } \\
\left(\operatorname{erg~cm}^{-2} \mathrm{~s}^{-1}\right)\end{array}$ \\
\hline 57766.757 & 16.2 & Swift-XRT & $0.3-10$ & 1.4 & $<8.1 \times 10^{-3}$ & $<3.4 \times 10^{-13}$ \\
\hline 57771.524 & 21.0 & Swift-XRT & $0.3-10$ & 7.5 & $<2.0 \times 10^{-3}$ & $<8.5 \times 10^{-14}$ \\
\hline 57792.390 & 41.8 & Chandra-ACIS & $0.5-7$ & 9.86 & $\left(5.0_{-2.9}^{+4.8}\right) \times 10^{-4}$ & $\left(5.0_{-2.9}^{+4.7}\right) \times 10^{-15}$ \\
\hline
\end{tabular}

Note. Epochs are calculated since the Fermi/GBM trigger time of GRB 161228B (MJD 57750.552).

after $r$-band maximum light; see Section 2.1) and on MJD $57811.454(\approx+43$ days after $r$-band maximum light; see Section 2.1), respectively, compared to SN 1998bw. Also, in this case we have subtracted host galaxy light from the iPTF17cw spectrum. The amount of de-reddening used to match the SN 1998bw spectra to that of iPTF17cw is significantly less than what is needed for the earlier comparison described above, thus indicating that iPTF17cw had colors more similar to SN 1998bw at this later phase.

Based on the above, we can reasonably estimate the iPTF17cw photospheric velocity around maximum light to be similar to that of SN 1998bw, $\left(19,500_{-1000}^{+1700}\right) \mathrm{km} \mathrm{s}^{-1}$ (see e.g., Lyman et al. 2016; Modjaz et al. 2016; Cano et al. 2017, and references therein). Indeed, if we assume that the minimum observed around $6600 \AA$ in the DCT and Keck spectra is due to the $\mathrm{Si} 6355 \AA$ line, the estimated velocities are of $(15,600 \pm 2000) \mathrm{kms}^{-1}$ at $\approx+17$ days since maximum light (DCT spectrum), and $17,300 \pm 2900$ at $\approx+43$ days since maximum (Keck spectrum). These velocities are compatible with the ones we measure for the $\mathrm{Fe} 5169 \AA$ line, of $17,300 \pm 1500 \mathrm{~km} \mathrm{~s}^{-1}$ and $17,500 \pm 1800 \mathrm{~km} \mathrm{~s}^{-1}$, respectively, for the DCT and Keck spectra. We thus estimate the photospheric velocity of iPTF17cw as the weighted mean of the speed values derived from the Si $6355 \AA$ and Fe $5169 \AA$ lines, $v_{\text {phot,peak }} \approx 16.9 \times 10^{3} \mathrm{~km} \mathrm{~s}^{-1}$. Using this in Equation (2) of (Lyman et al. 2016),

$$
v_{\text {phot,peak }} \approx 16.9 \times 10^{3} \mathrm{~km} \mathrm{~s}^{-1}=\left(\frac{10 E_{K}}{3 M_{\mathrm{eje}}}\right)^{1 / 2},
$$

which implies

$$
\left(\frac{E_{K}}{10^{51} \mathrm{erg}}\right) \approx 2 \times\left(\frac{M_{\mathrm{eje}}}{M_{\odot}}\right) .
$$

Combining the above Equation with Equation (7), we find the following for iPTF17cw:

$$
\begin{gathered}
M_{\text {eje }} \approx 2 M_{\odot}, \\
E_{K} \approx 4 \times 10^{51} \mathrm{erg} .
\end{gathered}
$$

These values are comparable to $M_{\text {eje }} \approx 1.9 M_{\odot}$ and $E_{K} \approx$ $3.3 \times 10^{51} \mathrm{erg}$ found for SN 2009bb (see Table 5 in Lyman et al. 2016), while $S N 1998 \mathrm{bw}$ had a larger ejecta mass and kinetic energy $\left(M_{\text {eje }} \approx 4.4 M_{\odot}\right.$ and $\left.E_{K} \approx 9.9 \times 10^{51} \mathrm{erg}\right)$.

\subsection{Association with GRB $161228 B$}

The results from the spectral fits of GRB 161228B described in Section 2.5 imply that GRB161228B is a "typical" GRB in terms of Band function parameters of GBM-detected long GRBs, with values of $E_{\text {peak }}$, peak flux, and fluence in the $\approx 50-60$ th percentile (Gruber et al. 2014). If we assume that
GRB 161228B and iPTF17cw are indeed associated, as suggested by the compatibility of localizations and trigger/ discovery time (see Section 2.5), we can use the redshift of iPTF17cw to calculate the k-corrected energy release and peak luminosity of GRB $161228 \mathrm{~B}$ over the $1 \mathrm{keV}-10 \mathrm{MeV}$ band. The derived isotropic-equivalent energy release in $\gamma$-rays, $E_{\text {iso }}$, is $(2.3 \pm 0.6) \times 10^{50} \mathrm{erg}$ and the peak luminosity, $L_{\text {peak }}$, is $(1.8 \pm 0.2) \times 10^{49} \mathrm{erg} \mathrm{s}^{-1}$. These values are at least one order of magnitude lower than the average observed $E_{\text {iso }}$ and $L_{\text {peak }}$ values of long GRBs. In fact, we estimate that these values are within the first percentile of long GRBs observed by GBM (Gruber et al. 2014). Although we find that the energy and luminosity are not as low as those for GRB 980425, our results for GRB 161228B are consistent with the observations of other SN-associated GRBs such as GRB 031203 (Soderberg et al. 2004) and GRB 120422A (Melandri et al. 2012). We stress, in particular, the similarity with GRB 031203, which also appeared to be an event with a spectrum similar to cosmological GRBs, but with a much lower isotropic energy release (Sazonov et al. 2004).

We estimate the probability of a chance coincidence between GRB 161228B and iPTF17cw by calculating the rate of Fermi GRBs falling in a random $103 \mathrm{deg}^{2}$ patch of sky (as large as the $1 \sigma$ region of GRB 161228 B localization; see Figure 8 ). This rate is $\approx 0.05$ per month, after taking into account the combined statistical and systematic localization uncertainties. Thus, a chance coincidence between iPTF17cw and GRB 161228B within a time window of a month has a probability of $\approx 5 \%$.

In order to further test the compatibility of GRB $161228 \mathrm{~B}$ explosion time with that of iPTF17cw, we fit the $r / R$-band light curve of SN $1998 \mathrm{bw}$ to the (k-corrected) $R$-band light curve of iPTF17cw (for more details, see F. Taddia et al. 2017, in preparation). In the fitting process, we allow the light curve of SN 1998bw to be stretched in time and shifted in magnitude, until we minimized the residuals of the difference between the stretched SN 1998bw light curve and the light curve of iPTF17cw. This fitting procedure is similar to that outlined in Cano (2013) and takes advantage of the known explosion epoch of SN 1998bw. We obtain a good fit at an explosion epoch corresponding to $\approx \mathrm{MJD} 57751.9 \pm 2.6$, which is not incompatible with the trigger time of GRB 161228B (MJD 57750.552).

We conclude that the association between iPTF17cw and GRB $161228 B$ is plausible. In what follows, we thus set the explosion time of iPTF17cw to be the trigger time of GRB 161228B (MJD 57750.552).

\subsection{Properties of the Fastest (Radio-emitting) Ejecta}

The temporal evolution of the candidate radio counterpart to iPTF17cw is shown in Figures 7 and 9. In these figures, we set the time of explosion to the trigger time of GRB 161228B. As evident from Figure 7 , the $6 \mathrm{GHz}$ luminosity of iPTF17cw is $\approx 10 \times$ smaller than GRB $980425 /$ SN 1998 bw, and $\approx 5 \times$ smaller 


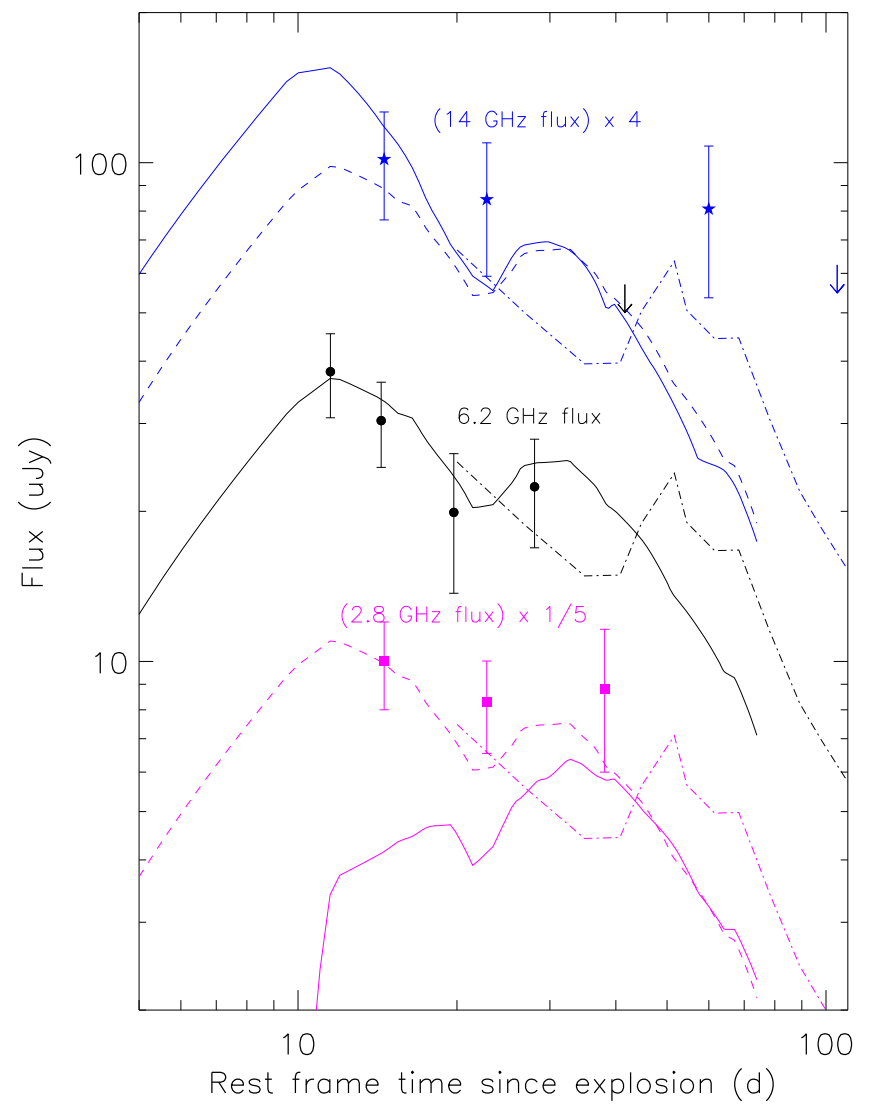

Figure 9. The multi-band $(2.8-14 \mathrm{GHz})$ radio light curves of iPTF17cw are shown and compared with the (interpolated) light curves of SN 1998bw at similar frequencies, scaled to match the luminosity of iPTF17cw (solid lines). The extrapolations of the scaled $5 \mathrm{GHz}$ light curve of SN $1998 \mathrm{bw}$ to $2.8 \mathrm{GHz}$ (magenta dashed line) and $14 \mathrm{GHz}$ (blue dashed line), using an optically thin spectral index of $\beta \approx-0.5$, are also shown. The $5 \mathrm{GHz}$ scaled light curve of SN 2009bb, and its extrapolations to 2.8 and $14 \mathrm{GHz}$ using the same spectral index of $\beta \approx-0.5$, are shown with dashed-dotted lines. See the text for a discussion.

than the relativistic SN 2009bb. The GHz radio luminosity of iPTF17cw is orders of magnitudes below the typical radio emission from cosmological GRBs (e.g., Chandra et al. 2012), but it is not incompatible with the radio emission observed from low-luminosity GRBs such as GRB 060218/SN 2006j and GRB 100316D/SN 2010bh. The radio spectral energy distribution suggests an optically thin spectrum around MJD 57766 $(\approx 16$ days since GRB 161228B). However, the measurement errors are large. Using the VLA multi-band observations collected around MJD 57766, we estimate a $2.8-14 \mathrm{GHz}$ spectral index $\left(f_{\nu} \propto \nu^{\beta}\right)$ of $\beta=-0.44 \pm 0.39$ around this epoch.

In Figure 9 we show the $2.8-14 \mathrm{GHz}$ radio light curves of iPTF17cw compared to the radio light curves of SN 1998bw at similar frequencies (solid lines), all scaled by a factor of $\approx 10$ in intrinsic luminosity to match the lower intrinsic luminosity of iPTF17cw (see Figure 7). Given the large distance to iPTF17cw, our radio measurements are affected by large errors. Thus, in what follows, we do not attempt a detailed modeling of iPTF17cw radio emission, but rather provide orders-of-magnitude estimates of the key properties of its radioemitting ejecta, based on comparisons with other radio SNe.

The $6 \mathrm{GHz}$ radio emission of iPTF17cw is qualitatively compatible with the double-peaked light curve of SN 1998bw, within the large measurement errors. However, a slowerevolving or later-peaking second radio peak, perhaps more similar to the one observed for SN $2009 \mathrm{bb}$ at $5 \mathrm{GHz}$ (dasheddotted lines), would be needed to account for the flat temporal behavior of the iPTF17cw counterpart at $14 \mathrm{GHz}$. For what concerns the emission at $2.8 \mathrm{GHz}$, we note that SN 1998bw emission was suppressed by synchrotron self-absorption (SSA) at the lower frequencies until $t \approx 40$ days since explosion (magenta solid line in Figure 9). Indeed, if we extrapolate the (scaled) $5 \mathrm{GHz}$ light curve of $\mathrm{SN} 1998 \mathrm{bw}$ to our other frequencies of interest $(2.8$ and $14 \mathrm{GHz})$ using an optically thin spectral index of $\beta \approx-0.5$ (dashed lines in Figure 9), we see that this extrapolation agrees well with the actual (scaled) light curves of SN 1998bw at $14 \mathrm{GHz}$ (blue solid line), while at $2.8 \mathrm{GHz}$ the agreement is good only at $t \gtrsim 40$ days (magenta dashed and solid lines). iPTF17 $\mathrm{cw}$ radio emission, on the other hand, appears to be optically thin at all frequencies $\gtrsim 2.8 \mathrm{GHz}$ already at $t \gtrsim 16$ days since explosion. In fact, the $2.8 \mathrm{GHz}$ emission of iPTF17cw is compatible with the optically thin extrapolation to $2.8 \mathrm{GHz}$ of the (scaled) $5 \mathrm{GHz}$ light curve of SN 1998bw (magenta dashed line in Figure 9). This, together with the fact that iPTF17cw emission appears to decrease with time at $6.2 \mathrm{GHz}$ over the first three epochs, suggests that the SSA peak frequency is $\nu_{p} \lesssim 2.8 \mathrm{GHz}$ at $\approx 16$ days since explosion (GRB 161228B), and thus $F_{p} \gtrsim 50 \mu \mathrm{Jy}$ (or $L_{p} \approx 10^{28} \mathrm{erg} \mathrm{s}^{-1} \mathrm{~Hz}^{-1}$ at the distance of iPTF17cw).

The above constraints on the SSA peak frequency and flux enable us to estimate the size of the radio-emitting material at $t \approx 16$ days since explosion. Using Equation (11) of Chevalier (1998):

$$
\begin{aligned}
R_{p} \approx & 6.6 \times 10^{15} \mathrm{~cm}\left(\frac{\eta}{2 \alpha}\right)^{1 / 17.2}\left(\frac{F_{p}}{\mathrm{Jy}}\right)^{8.1 / 17.2}\left(\frac{d_{L}}{\mathrm{Mpc}}\right)^{16.2 / 17.2} \\
& \times\left(\frac{\nu_{p}}{5 \mathrm{GHz}}\right)^{-1}
\end{aligned}
$$

where $\alpha \approx 1$ is the ratio of relativistic electron energy density to magnetic energy density, $F_{p}$ is the flux at the time of SSA peak, $\nu_{p}$ is the SSA frequency, and where we have parameterized with $\eta$ the thickness of the radiating electron shell (typically assumed to be in the range $2 \lesssim \eta \lesssim 10$; e.g., Soderberg et al. 2005; Chevalier \& Fransson 2006), and we assumed $p \approx 2.1$ for the power-law index of the electron energy distribution. Setting in the above Equation $F_{p} \gtrsim 50 \mu \mathrm{Jy}$ and $\nu_{p} \lesssim 2.8 \mathrm{GHz}$, we get $R_{p} \gtrsim 3.5 \times 10^{16}(\eta / 5)^{1 / 17.2} \mathrm{~cm}$ at 16 days since explosion, which implies that the average speed of the radio ejecta is $v_{s} \gtrsim\left[0.85 c(\eta / 5)^{1 / 17.2}\right]$, similarly to what was found for SN 2009bb (Soderberg et al. 2010). A more precise calculation for iPTF $17 \mathrm{cw}$ radio ejecta speed is obtained by considering the transverse shock speed constraint $\Gamma_{s} \beta_{s}$ $c=R_{p} / t \approx 0.85 c$, which implies $\Gamma_{s} \gtrsim 1.3$ or $\beta_{s} \gtrsim 0.65 c$.

The magnetic field can be expressed as (see Equation (12) in Chevalier 1998)

$$
B_{p} \approx 0.5\left(\frac{\eta}{2 \alpha}\right)^{4 / 17.2}\left(\frac{F_{p}}{\mathrm{Jy}}\right)^{-2 / 17.2}\left(\frac{d_{L}}{\mathrm{Mpc}}\right)^{-4 / 17.2}\left(\frac{\nu_{p}}{5 \mathrm{GHz}}\right) \mathrm{G}
$$




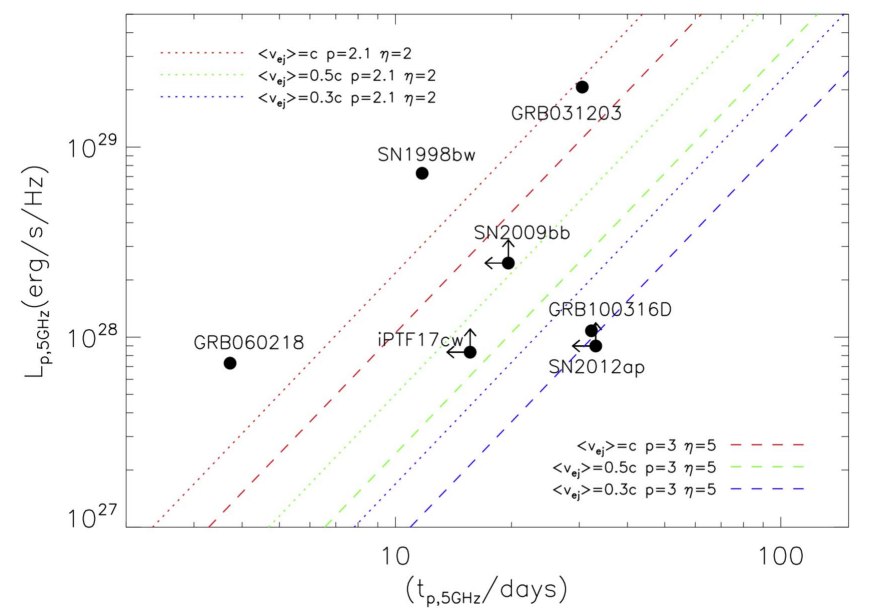

Figure 10. Constraints on the average (apparent) ejecta speed as derived using the $\approx 5 \mathrm{GHz}$ light curves shown in Figure 7 . These constraints represent lower limits to the ejecta speed for events like iPTF17cw, SN 2009bb, and SN 2012ap for which the SSA peak is already below $5 \mathrm{GHz}$ at the time of first observation. For low-luminosity GRBs, substantial deceleration of the fireball occurs at early times, so the speed constraints derived via this plot are lower limits to the initial speeds. Dotted lines are for $p=2.1$ and $\eta=2$, and dashed lines are for $p=3$ and $\eta=5$.

where we have set $p \approx 2.1$. The above gives $B_{p} \approx 0.3(\eta / 5)^{4 / 17.2}$ $\mathrm{G}$ at $t \approx 16$ days, for $\alpha \approx 0.1, F_{p} \approx 50 \mu \mathrm{Jy}$, and $\nu_{p} \approx 2.8 \mathrm{GHz}$.

We note that a lower limit on the average (apparent) speed of iPTF17cw ejecta can also be derived using our $6 \mathrm{GHz}$ observations only, and Equation (12). In fact, because the maximum flux measured at a given frequency is always smaller than or equal to the SSA peak flux, we can use the $6 \mathrm{GHz}$ light curve to set a lower limit on the ejecta speed (seem e.g., Berger et al. 2003a). We show this lower limit in Figure 10, together with similar constraints derived for the relativistic $\mathrm{SNe}$ and low-luminosity GRBs shown in Figure 7 (top panel). These represent reasonable (lower limit) estimates of the ejecta speeds derived in the literature via more complete modeling of the multi-frequency radio emission. The dashed and dotted lines in Figure 10 mark the range of speed constraints derived when the possible scatter in the values of $p$ and $\eta$ for different $\mathrm{SNe} /$ GRBs is taken into account.

Hereafter, we continue our discussion of the properties of the radio-emitting region of iPTFcw using a simple radiation model that neglects the dynamics (evolution of the radius $R$, magnetic field $B$, and minimum Lorentz factor of the accelerated electrons with time), and relativistic effects related to the high ejecta velocity. Based on the analysis of SN 1998bw by Li \& Chevalier (1999), we expect the corrections caused by dynamical and relativistic effects to be relatively modest, so our estimates below are likely within a factor of $\lesssim 2$ of the values that would be derived via a more detailed modeling.

For an SN shock expanding in a circumstellar medium (CSM) of density,

$$
\begin{aligned}
\rho & =5 \times 10^{11} \mathrm{~g} \mathrm{~cm}^{-1} A_{*} \times R^{-2} \\
& =\frac{\dot{M} /\left(10^{-5} M_{\odot} \mathrm{yr}^{-1}\right)}{4 \pi v_{w} /\left(10^{3} \mathrm{~km} \mathrm{~s}^{-1}\right)} \times R^{-2}
\end{aligned}
$$

assuming that a fraction $\epsilon_{B}$ of the energy density $\rho v_{s}^{2}$ goes into magnetic fields, one can write (see Equation (8) in Chevalier \&
Fransson 2006)

$$
\begin{aligned}
\frac{B_{p}^{2}}{8 \pi} \approx & 3 \times 10^{-2} A_{*}\left(\frac{\epsilon_{B}}{0.1}\right)\left(\frac{R_{p}}{3.5 \times 10^{16} \mathrm{~cm}}\right)^{-2} \\
& \times\left(\frac{v_{s}}{0.85 c}\right)^{2} \mathrm{erg} \mathrm{cm}^{-3} .
\end{aligned}
$$

From the above we get $A_{*} \approx 0.1(\eta / 5)^{8 / 17.2}$, or $\dot{M} \approx 10^{-6}$ $(\eta / 5)^{8 / 17.2} M_{\odot} \mathrm{yr}^{-1}$, for $\epsilon_{B} \approx 0.1$, and using the values of the shock radius and shock speed found previously. The total energy coupled to the fastest (radio-emitting) ejecta around $t \approx 16$ days can be expressed as

$$
E_{r} \approx \frac{4 \pi R_{p}^{3}}{\eta} \frac{B_{p}^{2}}{8 \pi \epsilon_{B}}=\frac{R_{p}^{3}}{\eta} \frac{B_{p}^{2}}{2 \epsilon_{B}}
$$

which yields $E_{r} \approx 4 \times 10^{48}(\eta / 5)^{11 / 17.2} \mathrm{erg}$ for $\epsilon_{B} \approx 0.1$, and using the value of the magnetic field found above. This energy is $\approx 0.1 \%$ of the total kinetic energy of the ejecta estimated via optical photometry (see Equation (11)).

The values of energy and CSM density found for iPTF17cw can be compared with $\dot{M} \approx 2.5 \times 10^{-7} M_{\odot} \mathrm{yr}^{-1}$ and $E_{r} \approx$ $(1-10) \times 10^{49}$ erg estimated for SN 1998bw by (Li \& Chevalier 1999), with $\dot{M} \approx 2 \times 10^{-6} M_{\odot} \mathrm{yr}^{-1}$ and $E_{r} \approx 1.3 \times$ $10^{49} \mathrm{erg}$ estimated for SN 2009bb (Soderberg et al. 2010), and with $\dot{M} \approx(0.4-1) \times 10^{-5} M_{\odot} \mathrm{yr}^{-1}$ and $E_{r} \approx(0.3-4) \times$ $10^{49}$ erg estimated for GRB 100316D (Margutti et al. 2013).

We finally note that because of the potential association with GRB 161228B, the early-time evolution of the radio (and X-ray counterparts) to iPTF17cw may be better described by that of a relativistic outflow that was originally aspherical. In this scenario, the relativistic ejecta are detached from the freely expanding SN outflow, decelerate, spread sideways, and become spherical on a timescale of the order of $t_{\text {sph }} \lesssim 6.2$ days $\times A_{*}^{-1}\left(E_{r} / 10^{49} \mathrm{erg}\right)$ (Waxman 2004). The last observation is smaller than the timescale of the earliest of our $2.8 \mathrm{GHz}$ observations of iPTF17cw for $\eta \gtrsim 5$. So our discussion (which assumes a spherical blastwave) is self-consistent for $\eta \gtrsim 5$.

\subsection{Radio Interpretation Caveats}

Because of the relatively large distance to iPTF $17 \mathrm{cw}$ $\left(d_{L} \approx 429 \mathrm{Mpc}\right)$, our most significant radio detections of this $\mathrm{SN}$ are at the $\approx 5 \sigma$ level. Thus, our radio measurements are affected by large errors, and so are the constraints on the spectral and temporal properties of the radio emission. Although the iPTF17cw $14 \mathrm{GHz}$ counterpart has faded below the detection level over the course of our follow-up, further VLA observations (in the A configuration, so as to reach better sensitivity and angular resolution) are needed to confirm fading at the lower frequencies. We were not able to carry out these observations due to the fact that the VLA moved to its most compact configuration (D config) over the course of our follow-up campaign. Because our conclusion that iPTF17cw is a relativistic event relies heavily on our first measurement at $2.8 \mathrm{GHz}$, hereafter we consider several factors that could contaminate the $2.8 \mathrm{GHz}$ flux.

In order to estimate what might be the contribution of background star formation to the measured $2.8 \mathrm{GHz}$ flux of iPTF17cw, pre-supernova photometry of the host galaxy was downloaded from the SDSS (Alam et al. 2015), the Galaxy 
Evolution Explorer (GALEX; Martin et al. 2005), and the Widefield Infrared Survey Explorer (Cutri et al. 2013); we also obtained images of the field from Pan-STARRS (Chambers et al. 2016) and performed aperture photometry of the host galaxy. We then performed a simple SED fit using the Bruzual \& Charlot (2003) stellar-population synthesis models and custom IDL software previously described in Perley et al. (2013). We infer a total stellar mass of $(3.5 \pm 0.3) \times 10^{9} M_{\odot}$ and a star formation rate (averaged over the past $50 \mathrm{Myr}$ ) of $0.31 \pm 0.05 M_{\odot} \mathrm{yr}^{-1}$, with no significant dust extinction. We note that these properties are quite similar to the LMC and to other GRB hosts at low redshift (e.g., Perley et al. 2016, and references therein). The host galaxy of iPTF17cw also shows a reddish concentration at its center, which could be a bulge or weak AGN. This level of star formation implies a total $1.4 \mathrm{GHz}$ spectral luminosity of the order of (Murphy et al. 2011)

$$
\left(\frac{L_{1.4 \mathrm{GHz}}}{\mathrm{erg} \mathrm{s}^{-1} \mathrm{~Hz}^{-1}}\right) \approx 1.57 \times 10^{28}\left(\frac{\mathrm{SFR}_{\text {radio }}}{M_{\odot} \mathrm{yr}^{-1}}\right) \approx 5 \times 10^{27}
$$

for the host galaxy of iPTFcw. Spectral indices of star-forming galaxies between 1.4 and $4.8 \mathrm{GHz}$ are estimated to be in the range $\approx-1$ to -0.4 (Seymour et al. 2008). Thus, we estimate the total radio emission from the host to be $\lesssim 4 \times 10^{27} \mathrm{erg} \mathrm{s}^{-1} \mathrm{~Hz}^{-1}$ at $2.8 \mathrm{GHz}$. Our first $2.8 \mathrm{GHz}$ observation of iPTF17cw implies a radio luminosity of $\approx 10^{28} \mathrm{erg}^{-1} \mathrm{~Hz}^{-1}$ concentrated in a compact region of FWHP size $\lesssim 0$ " 65 , much smaller than the $R$-band size of the host galaxy (see Figure 2). We thus consider a high level of contamination to the $\mathrm{PTF} 17 \mathrm{cw}$ radio counterpart from star formation in the host to be unlikely. Contamination from a compact radio source, i.e., an AGN, seems unlikely considering the fact that the optical, radio, and X-ray counterparts to iPTF17cw appear to be coincident and significantly offset from the host nucleus. Confirming fading of the radio counterpart of iPTF17cw at $2.8 \mathrm{GHz}$ in future observations will strengthen this conclusion.

We also explore possible effects related to interstellar scattering and scintillation (ISS). At the galactic coordinates of $\mathrm{iPTF} 17 \mathrm{cw} \quad\left(l \approx 178^{\circ}\right.$ and $\left.b \approx 42^{\circ}\right)$ the ISS transition frequency between the strong and weak scattering regimes is $\nu_{0} \approx 8 \mathrm{GHz}$, and the Fresnel angle at the transition frequency is $\Theta_{0} \approx 4 \mu$ as (Goodman 1997; Walker 2001). This is implies that our lowest-frequency observations are in the strong scattering regime. The modulation index in the refractive strong scattering reads

$$
\begin{gathered}
m(\nu) \approx\left(\frac{\Theta_{0}}{\Theta_{s}}\right)^{7 / 6}\left(\frac{\nu_{0}}{\nu}\right)^{2} \text { for } \Theta_{s}>\Theta_{r}, \\
m(\nu) \approx\left(\frac{\nu}{\nu_{0}}\right)^{17 / 30} \text { for } \Theta_{s}<\Theta_{r},
\end{gathered}
$$

where $\Theta_{r} \approx \Theta_{0}\left(\frac{\nu_{0}}{\nu}\right)^{11 / 5}$, and $\Theta_{s}$ is the source size. From the above, we see that the largest modulation index at $2.8 \mathrm{GHz}$ is $m \approx 0.55$ for a source of size $\Theta_{s}<\Theta_{r} \approx 40 \mu$ as.

To estimate whether ISS could conspire to invalidate our assumption of optically thin emission at $2.8 \mathrm{GHz}$ at 16 days since explosion, we calculate the modulation index that would be needed to enhance potentially optically thick emission at $2.8 \mathrm{GHz}$ to a level comparable to the one observed at day 16 (so that the $2.8-5 \mathrm{GHz}$ emission would appear optically thin, while being optically thick in reality):

$$
\begin{aligned}
& m(2.8 \mathrm{GHz}) \\
& \approx \frac{\sqrt{\left(f_{2.8 \mathrm{GHz}, 16 \text { days }}-f_{\text {thick, } 16 \text { days }}\right)^{2}-\left(\Delta f_{2.8 \mathrm{GHz}, 16 \text { days }}\right)^{2}}}{f_{2.8 \mathrm{GHz}, 16 \text { days }}},
\end{aligned}
$$

where $f_{\text {thick }} \approx 1.5 \times f_{6.2 \mathrm{GHz}} \times(2.8 \mathrm{GHz} / 6.2 \mathrm{GHz})^{5 / 2} \approx 6 \mu \mathrm{Jy}$ at day 16 (see Chevalier 1998, for the 1.5 correction factor applied here); $f_{2.8 \mathrm{GHz}, 16 \text { days }}$ is the measured flux at $2.8 \mathrm{GHz}$ on day 16 , and $\Delta f_{2.8 \mathrm{GHz}, 16 \text { days }}$ is its measurement error. We get $m$ $(2.8 \mathrm{GHz}) \approx 0.85$, which is larger than the largest possible modulation index at $2.8 \mathrm{GHz} m \approx 0.55$.

We thus conclude that iPTF17cw is a plausible relativistic SN candidate. However, we stress once again that re-observing the iPTF17cw field when the VLA will be back in its most extended configuration (2018 March), and confirming that the iPTF17cw radio counterpart has indeed faded below detection threshold at all frequencies (not only at $14 \mathrm{GHz}$, which we have already verified), will greatly help enhance the confidence in this interpretation.

\subsection{X-Ray Emission Modeling}

Our Chandra detection of iPTF17cw around day 40 since GRB 161228B implies an X-ray luminosity of $\approx(4-19) \times$ $10^{40} \mathrm{erg} \mathrm{s}^{-1}$, which is within the range observed at a similar epoch for the low-luminosity GRBs 980425 and 031203, most similar to the X-ray luminosity of GRB 100316D (Figure 7, bottom), $\gtrsim 10 \times$ larger than the X-ray luminosity of SN 2009bb at a similar epoch (Soderberg et al. 2010), and above the X-ray luminosity upper limit of $\approx 2.4 \times 10^{39} \mathrm{erg} \mathrm{s}^{-1}$ set by Chandra observations of SN 2012ap (Margutti et al. 2014). Because the radio luminosity of $\mathrm{PPTF} 17 \mathrm{cw}$ is smaller than that of GRB 980425 and GRB 031203 (see Figure 7), the comparable X-ray luminosity implies a flatter spectral index between the radio and X-ray bands. This is not unseen and links iPTF17cw to the low-luminosity GRBs 060218 and 100316D, for which an unusually flat radio-toX-ray spectral index was also observed (see Margutti et al. 2013, for a discussion).

From the ratio of the radio-to-X-ray (monochromatic) fluxes measured at the time of the Chandra observation $\left(F_{1 \mathrm{keV}} \approx 2 \times\right.$ $10^{-15} \mathrm{erg} \mathrm{cm}^{-2} \mathrm{~s}^{-1} \mathrm{keV}^{-1}$ ), we derive $\beta_{\text {radio- } \mathrm{X}} \sim-0.6$, which is compatible with the spectral index measured in the radio band, within the large errors (see Section 3.4). (We note that the extrapolation of the measured radio flux to the optical band with a similar spectral index yields an optical flux well below the one measured from iPTF17cw optical SN light curve.) The fact that the X-ray emission lies on the extrapolation from the radio band suggests a common origin for the radio and X-rays, likely nonthermal synchrotron emission from the shocked CSM, which is usually invoked in GRB afterglows and in several radio and Xray-emitting core-collapse SNe (Chevalier \& Fransson 2006). We note, however, that because of the large errors that affect our radio spectral index measurement, and because of the lack of a spectral index measurement in the X-rays, we cannot exclude that, similar to GRB $100316 \mathrm{D}$, the X-ray emission from iPTF17cw may be 
related to a different emission component (such as inverse Compton or prolonged central engine activity).

Within the synchrotron scenario, we can constrain the synchrotron cooling frequency to be $\nu_{c}(42$ days $) \gtrsim 1 \mathrm{keV}$ for iPTF17cw, due to the apparent lack of a spectral steepening between the radio and X-rays. Using Equation (11) of Chevalier \& Fransson (2006) (where we correct the normalization constant, erroneously reported there as $10^{10} \mathrm{GHz}$ to $\left.10^{10} \mathrm{~Hz}\right)$ :

$$
\nu_{c} \approx 8 \times 10^{12}\left(\frac{\epsilon_{B}}{0.1}\right)^{-3 / 2} A_{*}^{-3 / 2}\left(\frac{t}{42 \text { days }}\right) \mathrm{Hz} .
$$

From the above equation it is evident that $\nu_{c} \gtrsim 1 \mathrm{keV}$ would require an unusually low value of $A_{*}$. The lack of a spectral steepening at high frequencies is common to several other SN, including the radio-loud BL-Ic PTF11qcj (Corsi et al. 2014), SN 2009bb, and SN 1998bw, and may be explained by invoking a cosmic-ray-dominated $\mathrm{SN}$ shock (Ellison et al. 2000; Chevalier \& Fransson 2006).

We consider in what follows other possible sources of $\mathrm{X}$-rays generally invoked in the study of stripped-envelope core-collapse SNe (Chevalier \& Fransson 2006), such as thermal emission associated with the shock-heated ejecta, and inverse Compton (IC) up-scattering of optical photons by radio-emitting electrons.

As discussed in Corsi et al. (2014), within the thermal emission hypothesis, X-rays are produced while the forward shock plows into the CSM and/or by the reverse shock heating the ejecta. Thus, the X-ray luminosity depends on the density of the emitting material, which cools by free-free emission. Because the X-ray luminosity is directly proportional to the square of the mass-loss rate (see, e.g., Equation (16) in Corsi et al. 2014), for an event like iPTF17cw $\left(\approx 10^{-6} M_{\odot} \mathrm{yr}^{-1}\right)$ we don't expect free-free emission to be the dominant contributor to the iPTF17cw X-ray counterpart detected by Chandra.

The IC mechanism is expected to be most important on timescales comparable to the optical SN maximum, since the ratio of flux measured in the radio and X-ray bands is given by

$$
\frac{F_{X}}{\nu_{\text {radio }} F_{\nu, \text { radio }}} \sim \frac{U_{\mathrm{ph}}}{U_{B}}
$$

where $U_{\mathrm{ph}}$ is the energy density in up-scattered photons, which can be estimated as (Björnsson \& Fransson 2004; Soderberg et al. 2006b)

$$
U_{\mathrm{ph}} \approx 0.4 \times\left(\frac{L_{\mathrm{bol}}}{10^{42} \mathrm{erg} \mathrm{s}^{-1}}\right)\left(\frac{t}{1 \mathrm{day}}\right)^{-2}\left(\frac{v_{s}}{c}\right)^{-2} \mathrm{erg} \mathrm{cm}^{-3} .
$$

For iPTF17cw, even setting $L_{\text {bol }}$ to its peak value of $L_{\text {bol,peak }} \approx$ $10^{43} \mathrm{erg} \mathrm{s}^{-1}$ (at $t \approx 18$ days since GRB 161228B), and using $v_{s} \approx$ $0.85 c$ (see Section 3.4), we estimate $U_{\mathrm{ph}} \lesssim 2 \times 10^{-2} \mathrm{erg} \mathrm{cm}^{-3}$. From our radio observations, we also derive $\nu_{\text {radio }} F_{\nu \text {,radio }} \lesssim$ $2 \times 10^{-18} \mathrm{erg} \mathrm{cm}^{-2} \mathrm{~s}^{-1}$. Setting $B \sim 0.3$ ( $t / 16$ days $)^{-1} \mathrm{G}$ based on what we derived in Section 3.4 and assuming a typical $B \propto t^{-1}$ evolution of the magnetic field (Chevalier 1998), we derive the conservative upper limit

$$
F_{X}(42 \text { days }) \lesssim 10^{-16} \mathrm{erg} \mathrm{cm}^{-2} \mathrm{~s}^{-1},
$$

which is a factor of $\approx 10$ below the $\mathrm{X}$-ray flux measured by Chandra at $t \approx 42$ days since GRB 161228B.

We thus conclude that a synchrotron origin from a cosmicray-dominated shock is a more likely scenario for the radio and X-ray emission of iPTF17cw. However (as discussed above), we cannot exclude other mechanisms for the origin of the X-ray emission, such as long-lasting activity of the explosion central engine (e.g., a black-hole-plus-torus system, or a magnetar progenitor; Margutti et al. 2013). Modeling iPTF17cw emission within these more complex scenarios is (given our limited data set) beyond the scope of this paper.

\section{Summary and Conclusion}

We have presented the discovery, classification, and radioto-X-ray follow-up observations of iPTF17cw, a BL-Ic SN discovered by the iPTF while carrying out an extensive followup campaign of the (unrelated) Advanced LIGO event GW 170104. iPTF17cw spectroscopic and photometric properties show similarities with the engine-driven SN 1998bw and the relativistic SN 2009bb. We estimate a nickel mass similar to that of SN 1998bw, while the total energies of the ejecta and ejecta mass are more similar to those of SN 2009bb.

Our radio data suggest that $\mathrm{PTF} 17 \mathrm{cw}$ is a new member of the rare sample of relativistic $\mathrm{SNe}$ discovered independently of a $\gamma$-ray trigger. Given the temporal and spatial compatibility of iPTF17cw with GRB 161228B, we also consider iPTF17cw a potential new member of the limited sample of GRBassociated (engine-driven) BL-Ic SNe. We caution, however, that our radio observations are affected by large errors (given the large distance to iPTF17cw). Thus, further late-time VLA follow-up observations with high resolution are warranted to confirm the relativistic nature of iPTF17cw.

Radio-to-X-ray follow-up observations of iPTF17cw enabled us to estimate the properties of the fastest moving ejecta and those of the CSM. The radio luminosity of iPTF17cw is $\approx 10$ times smaller that SN 1998bw, but comparable to that of GRB 100316D. The fraction of energy coupled to the fastest emitting material is $\gtrsim 10$ times smaller than in SN 1998bw, while the CSM density around iPTF17cw is similar to that found around other relativistic BL-Ic SNe, such as SN 2009bb, and is consistent with values inferred for long GRBs. The relatively bright X-ray emission is compatible with the extrapolation from the radio band within a synchrotron emission scenario, and the implied X-ray luminosity is most similar to that of GRB 100316D.

Thanks to the increased rate of iPTF discoveries of BL-Ic $\mathrm{SNe}$, we have just now begun to observationally constrain the fraction $f$ of BL-Ic SNe that may be powered by central engines. Considering the size of the sample of BL-Ic SNe with radio observations we have collected so far, the discovery of iPTF17cw independently of a $\gamma$-ray trigger fits our expectations for $30 \% \lesssim f \lesssim 40 \%$ (Podsiadlowski et al. 2004; Corsi et al. 2016). This result also demonstrates that our discovery rate of engine-driven BL-Ic is likely to be substantially boosted by the advent of the Zwicky Transient Facility (ZTF; Smith et al. 2014; Bellm 2016). In the ZTF era, we expect to collect in one year a sample of BL-Ic SNe with radio observations as large as the one we collected with the iPTF in five years, and the discovery of events like iPTF17cw will be greatly facilitated.

A.C. and N.T.P. acknowledge support from the National Science Foundation under CAREER Grant No. 1455090. A.C. 
and N.T.P. also acknowledge partial support from the Swift Cycle 12 GI program (Grant No. NNX17AF93G). Support for this work was in part provided by the National Aeronautics and Space Administration through Chandra Award No. 19500451 issued by the Chandra X-ray Observatory Center, which is operated by the Smithsonian Astrophysical Observatory for and on behalf of the National Aeronautics Space Administration under contract NAS8-03060. This work was also supported by the GROWTH project funded by the National Science Foundation under grant No. 1545949. The National Radio Astronomy Observatory is a facility of the National Science Foundation operated under cooperative agreement by Associated Universities, Inc. These results made use of the Discovery Channel Telescope at Lowell Observatory. Lowell is a private, non-profit institution dedicated to astrophysical research and public appreciation of astronomy and operates the DCT in partnership with Boston University, the University of Maryland, the University of Toledo, Northern Arizona University, and Yale University. The upgrade of the DeVeny optical spectrograph has been funded by a generous grant from John and Ginger Giovale. This work made use of data supplied by the UK Swift Science Data Centre at the University of Leicester. This work is partially based on observations collected with the Liverpool Telescope, which is operated on the island of La Palma by Liverpool John Moores University in the Spanish Observatorio del Roque de los Muchachos of the Instituto de Astrofisica de Canarias with financial support from the UK Science and Technology Facilities Council. The PanSTARRS1 Surveys (PS1) have been made possible through contributions of the Institute for Astronomy, the University of Hawaii, the Pan-STARRS Project Office, the Max-Planck Society and its participating institutes, the Max-Planck Institute for Astronomy, Heidelberg and the Max-Planck Institute for Extraterrestrial Physics, Garching, The Johns Hopkins University, Durham University, the University of Edinburgh, Queen's University Belfast, the Harvard-Smithsonian Center for Astrophysics, the Las Cumbres Observatory Global Telescope Network Incorporated, the National Central University of Taiwan, the Space Telescope Science Institute, the National Aeronautics and Space Administration under grant No. NNX08AR22G issued through the Planetary Science Division of the NASA Science Mission Directorate, the National Science Foundation under grant No. AST-1238877, the University of Maryland, and Eotvos Lorand University (ELTE).

\section{ORCID iDs}

A. Corsi (1) https://orcid.org/0000-0001-8104-3536

S. B. Cenko (1) https://orcid.org/0000-0003-1673-970X

M. M. Kasliwal (1) https://orcid.org/0000-0002-5619-4938

R. Quimby (1) https://orcid.org/0000-0001-9171-5236

S. R. Kulkarni (1) https://orcid.org/0000-0001-5390-8563

A. M. Goldstein (i) https://orcid.org/0000-0002-0587-7042

N. Blagorodnova (1) https://orcid.org/0000-0003-0901-1606

D. A. Perley (1) https://orcid.org/0000-0001-8472-1996

L. P. Singer (1) https://orcid.org/0000-0001-9898-5597

C. Fremling (i) https://orcid.org/0000-0002-4223-103X

T. Kupfer (1) https://orcid.org/0000-0002-6540-1484

I. A. Steele (1) https://orcid.org/0000-0001-8397-5759

H. Vedantham (1) https://orcid.org/0000-0002-0872-181X

A. Kutyrev (i) https://orcid.org/0000-0002-2715-8460

N. T. Palliyaguru (i) https://orcid.org/0000-0002-4828-0262
J. Sollerman (1) https://orcid.org/0000-0003-1546-6615

E. Troja (i) https://orcid.org/0000-0002-1869-7817

S. Veilleux (1) https://orcid.org/0000-0002-3158-6820

\section{References}

Abbott, B., Abbott, R., Abbott, T., et al. 2017, PhRvL, 118, 221101 Alam, S., Albareti, F. D., Allende Prieto, C., et al. 2015, ApJS, 219, 12 Arnett, W. D. 1982, ApJ, 253, 785

Bellm, E. C. 2016, PASP, 128, 084501

Berger, E., Kulkarni, S. R., Frail, D. A., \& Soderberg, A. M. 2003a, ApJ, 599, 408

Berger, E., Kulkarni, S. R., Pooley, G., et al. 2003b, Natur, 426, 154

Bessell, M. S. 1999, PASP, 111, 1426

Bietenholz, M. F., De Colle, F., Granot, J., Bartel, N., \& Soderberg, A. M. 2014, MNRAS, 440, 821

Björnsson, C.-I., \& Fransson, C. 2004, ApJ, 605, 823

Bruzual, G., \& Charlot, S. 2003, MNRAS, 344, 1000

Burrows, D. N., Hill, J. E., Nousek, J. A., et al. 2005, SSRv, 120, 165

Campana, S., Mangano, V., Blustin, A. J., et al. 2006, Natur, 442, 1008

Cano, Z. 2013, MNRAS, 434, 1098

Cano, Z., Wang, S.-Q., Dai, Z.-G., \& Wu, X.-F. 2017, AdAst, 2017, 8929054

Cao, Y., Nugent, P. E., \& Kasliwal, M. M. 2016, PASP, 128, 114502

Cenko, S. B., Fox, D. B., Moon, D., et al. 2006, PASP, 118, 1396

Cenko, S. B., Kulkarni, S. R., Horesh, A., et al. 2013, ApJ, 769, 130

Cenko, S. B., Urban, A. L., Perley, D. A., et al. 2015, ApJL, 803, L24

Chakraborti, S., Soderberg, A., Chomiuk, L., et al. 2015, ApJ, 805, 187

Chambers, K. C., Magnier, E. A., Metcalfe, N., et al. 2016, arXiv:1612.05560

Chandra, P., Chevalier, R. A., Chugai, N., et al. 2012, ApJ, 755, 110

Chevalier, R. A. 1998, ApJ, 499, 810

Chevalier, R. A., \& Fransson, C. 2006, ApJ, 651, 381

Chomiuk, L., \& Soderberg, A. 2010, ATel, 2483, 1

Clocchiatti, A., Suntzeff, N. B., Covarrubias, R., \& Candia, P. 2011, AJ, 141, 163

Corsi, A., Gal-Yam, A., Kulkarni, S. R., et al. 2016, ApJ, 830, 42

Corsi, A., Kasliwal, M., Cenko, S., et al. 2017, GCN, 20364, https://gcn.gsfc. nasa.gov/other/G268556.gcn3

Corsi, A., Ofek, E. O., Frail, D. A., et al. 2011, ApJ, 741, 76

Corsi, A., Ofek, E. O., Gal-Yam, A., et al. 2014, ApJ, 782, 42

Currie, M. J., Berry, D. S., Jenness, T., et al. 2014, in ASP Conf. Ser. 485 Astronomical Data Analysis Software and Systems XXIII, ed. N. Manset \& P. Forshay (San Francisco, CA: ASP), 391

Cutri, R. M., Wright, E. L., Conrow, T., et al. 2013, yCat, 2328

Dickey, J. M., \& Lockman, F. J. 1990, ARA\&A, 28, 215

Drake, A. J., Djorgovski, S. G., Mahabal, A. A., et al. 2013, ATel, 4984, 1

Ellison, D. C., Berezhko, E. G., \& Baring, M. G. 2000, ApJ, 540, 292

Evans, P., Corsi, A., Kennea, J., et al. 2017, LIGO-Virgo GRB Coordinate Network Circular, 20473, https://gcn.gsfc.nasa.gov/other/G268556.gcn3 Evans, P. A., Beardmore, A. P., Page, K. L., et al. 2009, MNRAS, 397, 1177 Filippenko, A. V. 1982, PASP, 94, 715

Frail, D. A., Soderberg, A. M., Kulkarni, S. R., et al. 2005, ApJ, 619, 994 Fremling, C., Sollerman, J., Taddia, F., et al. 2016, A\&A, 593, A68

Galama, T. J., Vreeswijk, P. M., van Paradijs, J., et al. 1998, Natur, 395, 670 Gehrels, N., Chincarini, G., Giommi, P., et al. 2005, ApJ, 621, 558

Goodman, J. 1997, NewA, 2, 449

Gruber, D., Goldstein, A., Weller von Ahlefeld, V., et al. 2014, ApJS, 211, 12 Högbom, J. A. 1974, A\&AS, 15, 417

Horne, K. 1986, PASP, 98, 609

Howell, D. A., Sullivan, M., Nugent, P. E., et al. 2006, Natur, 443, 308 Iwamoto, K., Mazzali, P. A., Nomoto, K., et al. 1998, Natur, 395, 672

Kaiser, N., Burgett, W., Chambers, K., et al. 2010, Proc. SPIE, 7733, 77330E Kamble, A., \& Soderberg, A. 2013, ATel, 4997, 1

Kasliwal, M., Singer, L., Karamehmetoglu, E., et al. 2017, LIGO-Virgo GRB Coordinate Network Circular, 20398, https://gcn.gsfc.nasa.gov/other/ G268556.gen3

Kelson, D. D. 2003, PASP, 115, 688

Kouveliotou, C., Woosley, S. E., Patel, S. K., et al. 2004, ApJ, 608, 872

Kulkarni, S. R., Frail, D. A., Wieringa, M. H., et al. 1998, Natur, 395, 663

Kupfer, T., Quimby, R., Adams, S., Vedantham, H., \& Kasliwal, M. 2017, LIGO-Virgo GRB Coordinate Network Circular, 20419, https://gcn.gsfe. nasa.gov/gcn3/20419.gcn3

Law, N. M., Kulkarni, S. R., Dekany, R. G., et al. 2009, PASP, 121, 1395

Li, Z.-Y., \& Chevalier, R. A. 1999, ApJ, 526, 716

LIGO Scientific Collaboration,Virgo Collaboration 2017a, LIGO-Virgo GRB Coordinate Network Circular, 20364, https://gcn.gsfc.nasa.gov/other/ G268556.gen3 
LIGO Scientific Collaboration,Virgo Collaboration 2017b, LIGO-Virgo Circular, 20385, https://gcn.gsfc.nasa.gov/other/G268556.gcn3

LIGO Scientific Collaboration,Virgo Collaboration 2017c, LIGO-Virgo GRB Coordinate Network Circular, 21056, https://gcn.gsfc.nasa.gov/other/ G268556.gcn3

Lyman, J. D., Bersier, D., \& James, P. A. 2014, MNRAS, 437, 3848

Lyman, J. D., Bersier, D., James, P. A., et al. 2016, MNRAS, 457, 328

Marcinkowski, R., Xiao, H., \& Hajdas, W. 2016, GCN, 20348, 1

Margutti, R., Milisavljevic, D., Soderberg, A. M., et al. 2014, ApJ, 797, 107

Margutti, R., Soderberg, A. M., Wieringa, M. H., et al. 2013, ApJ, 778, 18

Martin, D. C., Fanson, J., Schiminovich, D., et al. 2005, ApJL, 619, L1

Masci, F. J., Laher, R. R., Rebbapragada, U. D., et al. 2017, PASP, 129, 014002

McMullin, J. P., Waters, B., Schiebel, D., Young, W., \& Golap, K. 2007, in

ASP Conf. Ser. 376, Astronomical Data Analysis Software and Systems

XVI, ed. R. A. Shaw, F. Hill, \& D. J. Bell (San Francisco, CA: ASP), 127

Melandri, A., Pian, E., Ferrero, P., et al. 2012, A\&A, 547, A82

Milisavljevic, D., Margutti, R., Parrent, J. T., et al. 2015, ApJ, 799, 51

Modjaz, M., Liu, Y. Q., Bianco, F. B., \& Graur, O. 2016, ApJ, 832, 108

Murphy, E. J., Condon, J. J., Schinnerer, E., et al. 2011, ApJ, 737, 67

Ofek, E. O., Cenko, S. B., Gal-Yam, A., et al. 2007, ApJ, 662, 1129

Ofek, E. O., Laher, R., Law, N., et al. 2012, PASP, 124, 62

Oke, J. B., Cohen, J. G., Carr, M., et al. 1995, PASP, 107, 375

Oke, J. B., \& Gunn, J. E. 1982, PASP, 94, 586

Perley, D. A., Cenko, S. B., Corsi, A., et al. 2014, ApJ, 781, 37

Perley, D. A., Levan, A. J., Tanvir, N. R., et al. 2013, ApJ, 778, 128

Perley, D. A., Tanvir, N. R., Hjorth, J., et al. 2016, ApJ, 817, 8
Piascik, A. S., Steele, I. A., Bates, S. D., et al. 2014, Proc. SPIE, 9147, 91478H Pignata, G., Stritzinger, M., Soderberg, A., et al. 2011, ApJ, 728, 14

Podsiadlowski, P., Mazzali, P. A., Nomoto, K., Lazzati, D., \& Cappellaro, E. 2004, ApJL, 607, L17

Rau, A., Kulkarni, S. R., Law, N. M., et al. 2009, PASP, 121, 1334

Richmond, M. W., van Dyk, S. D., Ho, W., et al. 1996, AJ, 111, 327

Salas, P., Bauer, F. E., Stockdale, C., \& Prieto, J. L. 2013, MNRAS, 428, 1207

Sazonov, S. Y., Lutovinov, A. A., \& Sunyaev, R. A. 2004, Natur, 430, 646

Schlafly, E. F., \& Finkbeiner, D. P. 2011, ApJ, 737, 103

Seymour, N., Dwelly, T., Moss, D., et al. 2008, MNRAS, 386, 1695

Singer, L. P., Kasliwal, M. M., Cenko, S. B., et al. 2015, ApJ, 806, 52

Smith, R. M., Dekany, R. G., Bebek, C., et al. 2014, Proc. SPIE, 9147, 914779

Soderberg, A., \& Chomiuk, L. 2011, ATel, 3101, 1

Soderberg, A. M., Chakraborti, S., Pignata, G., et al. 2010, Natur, 463, 513

Soderberg, A. M., Kulkarni, S. R., Berger, E., et al. 2004, Natur, 430, 648

Soderberg, A. M., Kulkarni, S. R., Berger, E., et al. 2005, ApJ, 621, 908

Soderberg, A. M., Kulkarni, S. R., Nakar, E., et al. 2006a, Natur, 442, 1014

Soderberg, A. M., Nakar, E., Berger, E., \& Kulkarni, S. R. 2006b, ApJ, 638,930

Wade, R. A., \& Horne, K. 1988, ApJ, 324, 411

Walker, M. A. 2001, MNRAS, 321, 176

Watson, D., Hjorth, J., Levan, A., et al. 2004, ApJL, 605, L101

Waxman, E. 2004, ApJ, 602, 886

Woosley, S. E., \& Bloom, J. S. 2006, ARA\&A, 44, 507

Yaron, O., \& Gal-Yam, A. 2012, PASP, 124, 668

York, D. G., Adelman, J., Anderson, J. E., Jr., et al. 2000, AJ, 120, 1579 